Mon. Not. R. Astron. Soc. 000, 000-000 (0000) Printed 21 October $2018 \quad$ (MN LATEX style file v2.2)

\title{
Deuterium enrichment of the interstellar grain mantle
}

\author{
Ankan Das ${ }^{1}$, Dipen Sahu ${ }^{1}$, Liton Majumdar ${ }^{2,3,1}$, Sandip K. Chakrabarti ${ }^{4,1}$ \\ ${ }^{1}$ Indian Centre for Space Physics, Chalantika 43, Garia Station Rd., Kolkata, 700084, India \\ 2 Univ. Bordeaux, LAB, UMR 5804, F-33270, Floirac, France \\ 3 CNRS, LAB, UMR 5804, F-33270, Floirac, France \\ ${ }^{4}$ S. N. Bose National Centre for Basic Sciences, Salt Lake, Kolkata 700098, India
}

\begin{abstract}
We carry out Monte-Carlo simulation to study deuterium enrichments of interstellar grain mantles under various physical conditions. Based on the physical properties, various types of clouds are considered. We find that in diffuse cloud regions, very strong radiation fields persists and hardly a few layers of surface species are formed. In translucent cloud regions with a moderate radiation field, significant number of layers would be produced and surface coverage is mainly dominated by photo-dissociation products such as, $\mathrm{C}, \mathrm{CH}_{3}, \mathrm{CH}_{2} \mathrm{D}, \mathrm{OH}$ and $\mathrm{OD}$. In the intermediate dense cloud regions (having number density of total hydrogen nuclei in all forms $\sim 2 \times 10^{4} \mathrm{~cm}^{-3}$ ), water and methanol along with their deuterated derivatives are efficiently formed. For much higher density regions $\left(\sim 10^{6} \mathrm{~cm}^{-3}\right)$, water and methanol productions are suppressed but surface coverages of $\mathrm{CO}, \mathrm{CO}_{2}, \mathrm{O}_{2}, \mathrm{O}_{3}$ are dramatically increased. We find a very high degree of fractionation of water and methanol. Observational results support a high fractionation of methanol but surprisingly water fractionation is found to be low. This is in contradiction with our model results indicating alternative routes for de-fractionation of water. Effects of various types of energy barriers are also studied. Moreover, we allow grain mantles to interact with various charged particles (such as $\mathrm{H}^{+}, \mathrm{Fe}^{+}, \mathrm{S}^{+}$and $\mathrm{C}^{+}$) to study the stopping power and projected range of these charged particles on various target ices.
\end{abstract}

Key words: Molecular clouds, ISM, abundances, molecules, chemical evolution, Monte-Carlo simulations

\section{INTRODUCTION}

Astronomical observations along with laboratory studies reveal the presence of numerous organic molecules in interstellar space (Herbst \& van Dishoeck 2009; Orgel 2004; Abelson 1966). Modeling of interstellar composition also supports indigenous formation of these molecules (Das et al. 2008a; Chakrabarti et al. 2015; Maiumdar et al. 2012, 2013; Das et al. 2015a). Modeling complements observational and laboratory studies by emphasizing the role of interstellar dusts towards the formation of these molecules. Mainly silicate and carbonaceous grains dominate in an interstellar space and they are thought to have molecular ice layers (Draine 2003; Gibb et al. 2004). In fact, even the huge abundance of molecular hydrogen could not be explained without invoking interstellar dusts (Biham et al. 2001; Chang. Cuppen \& Herbst 2005; Chakrabarti et al. 2006a. b; Sahu et al. 2015). It is now believed that at least some significant fraction of the interstellar species would be synthesized on interstellar dusts (Tielens \& Hagen 1982; Das, Acharvva \& Chakrabarti 2010; Das \& Chakrabarti 2011; Das et al. 2008b).
Stars are mainly formed in the densest part of an interstellar cloud called as pre-stellar core. These regions are extremely cold $(<10 \mathrm{~K})$ and dense $\left(>10^{5} \mathrm{~cm}^{-3}\right)$. At these temperatures and densities, gas phase species could stick to a grain surface and lighter species could migrate through the surface either by thermal hopping or by quantum mechanical tunnelling. Earlier studies for the formations of interstellar grain mantle by various authors (Stantcheva \& Herbst 2004; Das, Acharvva \& Chakrabarti 2010; Das \& Chakrabarti 2011) pointed out that water pointed out that water and methanol were mainly produced by this method. In the close vicinity of a forming protostar, much higher temperature and higher fluxes of various energetic particles induce evaporation of the grain mantle.

Fascinating progress about the study of the interstellar molecules in ice phase started after the launch of Infrared Space Observatory (ISO) with the Short Wavelength Spectrometer (SWS) instrument on board. It was found that water is dominating the mantle composition and could account for $60-70 \%$ of the ice in most of the lines of sight (Whittet 2003; Gibb et al. 2004). Since icy mantles are mainly dominated by water molecules, abundances of any 
Ankan Das, Dipen Sahu, Liton Majumdar, Sandip K. Chakrabarti

ice phase species mostly represented with respect to the ice phase water abundance. Other detected ice phase species are $\mathrm{CO}, \mathrm{CO}_{2}, \mathrm{OCS}, \mathrm{CH}_{3} \mathrm{OH}, \mathrm{CH}_{4}$ etc.. $\mathrm{OCN}^{-}$(Whittet et al. 2001) and $\mathrm{NH}_{4}^{+}$are supposed to be the carriers of some unidentified features as well.

Composition of interstellar grain mantle varies throughout their evolutionary stages depending upon the amount of processing of the icy mantle materials. Gibb et al. (2004) classified various sources into five categories namely, Quiescent environment (example: Elias 16), Low-mass YSO (example: Elias 29), intermediate-mass YSO (example: AFGL 989, R CrA IRS 1), High-mass YSO with weak processing (example: W3 IRS 5, Mon R2 IRS 2, Mon R2 IRS 3) and High mass YSO with strong processing (example: W33A, AFGL 7009S). Around the quiescent dark molecular cloud regions, they found polar $\left(\mathrm{H}_{2} \mathrm{O}, \mathrm{CO}\right.$ and possibly $\mathrm{NH}_{3}$ bearing) mantle coated by $\mathrm{CO}$ rich apolar mantle and trace amount of $\mathrm{CH}_{3} \mathrm{OH}$ and $\mathrm{XCN}$. $\mathrm{CO}$ and $\mathrm{CO}_{2}$ are found to be covered by $\sim 25 \%$ each with $\mathrm{NH}_{3}<10 \%$, Methanol $<3 \%$ and $\mathrm{XCN}<1.5 \%$. In case of Low-mass YSOs they found that $\mathrm{CO}_{2}$ is dominating $(\sim 20 \%)$, $\mathrm{CO}$ is $\sim 5 \%, \mathrm{NH}_{3}$ is $<5 \%, \mathrm{CH}_{3} \mathrm{OH}$ is $<3 \%$ and $\mathrm{XCN}$ is $<1.5 \%$. However, Pontoppidan et al. (2003) reported $\sim 15 \%$ abundance of methanol in various low mass YSOs. This suggests that production of methanol is not only limited to high mass YSOs. Intermediate-mass YSOs are found to be dominated by large amounts of $\mathrm{CO}_{2}(\sim 30-35 \%)$ and $\mathrm{CO}(18-50 \%)$ with trace amount of $\mathrm{NH}_{3}(<5 \%)$, methanol $(<5 \%)$ and $\mathrm{XCN}$ $(0.3 \%)$. High-mass YSOs with weak processing is found to be dominated by $\mathrm{CO}_{2}(7-22 \%)$ and methanol $(<5-10 \%)$. Minor components are $\mathrm{CO}(3-8 \%), \mathrm{NH}_{3}(<5 \%)$ and $\mathrm{XCN}$ $(0.3-2 \%)$. Around the High-mass YSOs with strong processing, ethanol, $\mathrm{CO}_{2}, \mathrm{CO}, \mathrm{NH}_{3}$ and $\mathrm{XCN}$ are found to be $15-30 \%, 13-23 \%, 8-17 \%, 15 \%, 2-6 \%$ respectively.

Despite low elemental abundances of atomic deuterium in interstellar space having average $\mathrm{D} / \mathrm{H}$ ratio of $1.5 \times 10^{-5}$ (Linsky et al. 1995, 2003; Stancil, Lepp \& Dalgarno 1998) in the ISM, several species are found to be heavily fractionated (Wakelam et al. 2014; Das et al. 2013b; Das et al. 2015b; Majumdar et al. 2014a.b). In molecular clouds, the $\mathrm{D}$ reservoir is HD. The starting point of the deuterium fractionation is the following reaction between $\mathrm{H}_{3}{ }^{+}$and $\mathrm{HD}$ which produces $\mathrm{H}_{2} \mathrm{D}^{+}$. This reaction can not follow the reverse step when kinetic temperature is less than $20 \mathrm{~K}$. Thus abundances of $\mathrm{H}_{2} \mathrm{D}^{+}$steadily increase and $\mathrm{H}_{2} \mathrm{D}^{+} / \mathrm{H}_{3}{ }^{+}$ratio become significantly higher than the $\mathrm{D}$ elemental abundance with respect to $\mathrm{H}$. $\mathrm{CO}$ and $\mathrm{O}$ are the major destruction partner of $\mathrm{H}_{2} \mathrm{D}^{+}$. Around the $\mathrm{CO}$ and $\mathrm{O}$ depleted region, deuterium fractionation is further enhanced and produce multiply deuterated $\mathrm{H}_{3}{ }^{+}$. Dissociative recombination of these multiply deuterated $\mathrm{H} 3+$ then produces $\mathrm{D}$ atoms and imply an atomic D/H ratio $>0.1$ (Caselli \& Ceccarelli 2012). This large atomic $\mathrm{D} / \mathrm{H}$ ratio in gas phase effectively reflects major deuteration of the surface species. It is to be noted that extreme fractionation have only been observed in Lowmass prestellar cores and protostars. Probably due to the warmer environments, massive protostars do not show the same deuterium enrichment (Ceccarelli et al. 2007).

As per the elemental $\mathrm{D} / \mathrm{H}$ ratio, singly deuterated species are expected to be $\sim 10^{5}$ times less abundant than their hydrogenated form. Similarly the doubly and triply deuterated forms are expected to be $\sim 10^{10}$ and $10^{15}$ times respectively lower. But in reality this is not be the case instead the deuterated ratios are found to be extremely higher with enhancements of the $\mathrm{D} / \mathrm{H}$ of up to 13 orders of magnitude with respect to the elemental $\mathrm{D} / \mathrm{H}$ abundance ratio (Caselli \& Ceccarelli 2012). Over the last few years multiply deuterated isotopologues of some common interstellar molecules, such as methanol, formaldehyde, thioformaldehyde, hydrogen sulfide and ammonia have been observed. Multiply deuterated formaldehydes have been observed around various sources. First doubly deuterated molecule detected in the ISM was D2CO. It was observed in Orion Compact Ridge (High-mas star forming regions) by Turner (1990). They found a fractionation value of $\sim 14 \%$ for singly deuterated formaldehyde and $\sim 0.3 \%$ for doubly deuterated formaldehyde. However, Loinard et al. (2001) found a fractionation value $\sim 40 \pm 20 \%$ for doubly deuterated formaldehyde towards the Low-mass protostar IRAS 16293E. Multiply deuterated ammonia molecules were observed during the last few years. Singly deuterated ammonia, $\mathrm{NH}_{2} \mathrm{D}$ bears the fractionation ratio of 0.1 in the class 0 source, NGC 1333 IRAS 4A van der Tak \& van Dishoeck 2000). Doubly deuterated form of ammonia was observed by Roueff et al. (2000) in a cold starless cloud, L134N having a fractionation ratio of 0.05 . Moreover, triply deuterated ammonia, $\mathrm{ND}_{3}$ was detected by van der Tak et al. (2002) in NGC 1333 IRAS 4A with a fractionation ratio 0.001. Deuterated methanols were detected along IRAS 16293 (Parise et al. 2002, 2004). They obtained the fractionation ratio for singly deuterated methanol $0.9 \pm 0.3$ and $0.04 \pm 0.02$ for $\mathrm{CH}_{2} \mathrm{DOH} / \mathrm{CH}_{3} \mathrm{OH}$ and $\mathrm{CH}_{3} \mathrm{OD} / \mathrm{CH}_{3} \mathrm{OH}$ respectively. In case of the doubly deuterated methanol $\left(\mathrm{CHD}_{2} \mathrm{OH} / \mathrm{CH}_{3} \mathrm{OH}\right)$, they derived a ratio of $0.2 \pm 0.1$. For the triply deuterated methanol $\left(\mathrm{CD}_{3} \mathrm{OH} / \mathrm{CH}_{3} \mathrm{OH}\right)$ they obtained a fractionation ratio averaged on a 10 inch beam is 0.014 .

Water is found to be comparatively less fractionated in comparison to methanol around various sources. It was observed that the deuterium fractionation of water can be about $\mathrm{HDO} / \mathrm{H}_{2} \mathrm{O} \sim 0.01 \%$ towards massive hot cores (Gensheimer, Mauersberger \& Wilson 1996) and $~ 0.02-$ $0.05 \%$ in comets and asteroids (Altwegg \& Bockelée-Morvan 2003). According to the Vienna Standard Mean Ocean Water value, the fractionation ratio of $\mathrm{HDO} / \mathrm{H}_{2} \mathrm{O}$ is $\sim 1.588 \pm$ $0.001 \times 10^{-4}$ in our oceans. However, some observations predicted a high fractionation ratio of a few $\%$ towards the hot corino NGC1333-IRAS2A (Liu et al. 2011), and IRAS16283-2422 (Parise et al. 2005). Butner et al. (2007) discovered doubly deuterated water toward the protostellar binary system IRAS 16293-2422. They derived $\mathrm{D}_{2} \mathrm{O} / \mathrm{H}_{2} \mathrm{O}$ ratio $5 \times 10^{-5}$ for the hot corino gas. Surface reactions play major role for the formation of water molecules in the ISM (Das \& Chakrabarti 2011; Cazaux et al. 2010). It is believed that it is most likely that the formation of water molecules on the surfaces of interstellar grains occurred before the formation of the solar nebula in the proto-solar molecular cloud (Jorgensen \& van Dishoeck 2010; Taquet et al. 2013). Isotopic composition of water along with the ortho-para ratio of the water formation epoch could be preserved in some objects like comets. Since ground base observation of water molecules are hindered by the huge abundance of water in the Earths atmosphere, various space based observations such as ISO, Spitzer, ODIN, SWAS, Her- 
Table 1. Initial gas phase abundances

\begin{tabular}{cc}
\hline Species & $\begin{array}{c}\text { Number density of cloud } \\
\left(10^{4} \mathbf{c m}^{-3}\right)\end{array}$ \\
\hline $\mathrm{H}$ & 1.1 \\
$\mathrm{D}$ & $1.1 r_{d}$ \\
$\mathrm{O}$ & 1.05 \\
$\mathrm{CO}$ & 0.15 \\
\hline
\end{tabular}

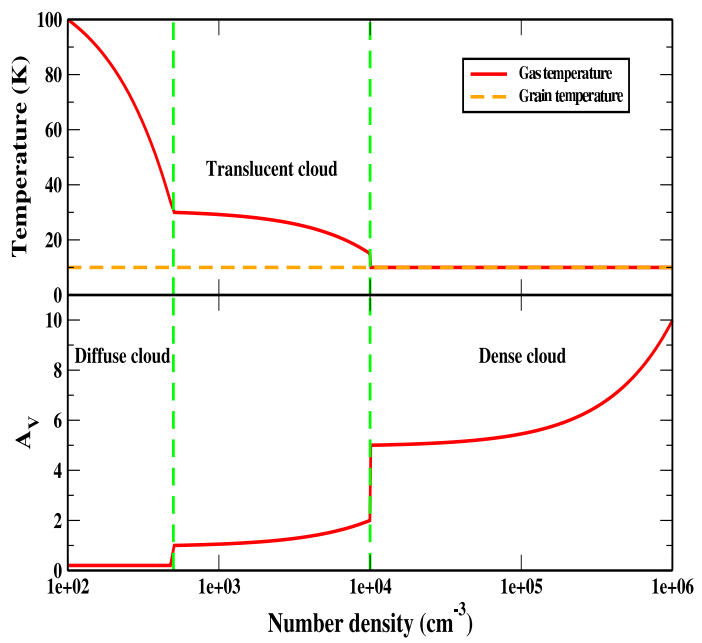

Figure 1. Various types of clouds based on $n_{H}, T_{g a s}$ and $A_{V}$.

schel etc. were carried out. Deuterium fractionation of water could be used as an important diagnostics tool to find out a connection between the sources of Earths water fractionation (which is believed to have been brought in by the comets) and interstellar water.

In this paper, we mainly focus on the deuterium enrichment of interstellar grain mantle under various conditions. In Section 2, modeling details are presented. In Section 3, we present our results and finally in Section 4, we draw our conclusions.

\section{MODELING DETAILS}

\subsection{Chemical modeling}

It is well known that Monte Carlo method is computation intensive since Monte Carlo codes needs to handle very different time-scales for different species. However, it gives the desired accuracy and the results are realistic. Here, we use continuous time random walk Monte Carlo method to study the chemical evolution of the grain mantle. This code was originally developed by (Das, Acharvva \& Chakrabarti 2010) and modified by Das \& Chakrabarti (2011). In order to study the deuterium enrichment of the grain mantle, we include some more reactions to this code. To save computational time, we consider $50 \times 50$ sites of grains (as in Das, Acharvva \& Chakrabarti (2010); Das \& Chakrabarti (2011)) and extrapolate the results for the classical grains
Table 2. Surface Reactions considered

\begin{tabular}{|c|c|c|}
\hline & Reactions & $\mathrm{E}_{a}(\mathrm{~K})$ \\
\hline 1 & $\mathrm{H}+\mathrm{H} \rightarrow \mathrm{H}_{2}$ & \\
\hline 2 & $\mathrm{H}+\mathrm{O} \rightarrow \mathrm{OH}$ & \\
\hline 3 & $\mathrm{H}+\mathrm{OH} \rightarrow \mathrm{H}_{2} \mathrm{O}$ & \\
\hline 4 & $\mathrm{H}+\mathrm{CO} \rightarrow \mathrm{HCO}$ & 390 \\
\hline 5 & $\mathrm{H}+\mathrm{HCO} \rightarrow \mathrm{H}_{2} \mathrm{CO}$ & \\
\hline 6 & $\mathrm{H}+\mathrm{H}_{2} \mathrm{CO} \rightarrow \mathrm{H}_{3} \mathrm{CO}$ & 415 \\
\hline 7 & $\mathrm{H}+\mathrm{H}_{3} \mathrm{CO} \rightarrow \mathrm{CH}_{3} \mathrm{OH}$ & \\
\hline 8 & $\mathrm{O}+\mathrm{O} \rightarrow \mathrm{O}_{2}$ & \\
\hline 9 & $\mathrm{O}+\mathrm{CO} \rightarrow \mathrm{CO}_{2}$ & 1000 \\
\hline 10 & $\mathrm{O}+\mathrm{HCO} \rightarrow \mathrm{CO}_{2}+\mathrm{H}$ & \\
\hline 11 & $\mathrm{O}+\mathrm{O}_{2} \rightarrow \mathrm{O}_{3}$ & \\
\hline 12 & $\mathrm{H}+\mathrm{O}_{2} \rightarrow \mathrm{HO}_{2}$ & \\
\hline 13 & $\mathrm{H}+\mathrm{HO}_{2} \rightarrow \mathrm{H}_{2} \mathrm{O}_{2}$ & \\
\hline 14 & $\mathrm{H}+\mathrm{H}_{2} \mathrm{O}_{2} \rightarrow \mathrm{H}_{2} \mathrm{O}+\mathrm{OH}$ & \\
\hline 15 & $\mathrm{H}+\mathrm{O}_{3} \rightarrow \mathrm{O}_{2}+\mathrm{OH}$ & 450 \\
\hline 16 & $\mathrm{H}_{2}+\mathrm{OH} \rightarrow \mathrm{H}_{2} \mathrm{O}+\mathrm{H}$ & 2600 \\
\hline 17 & $\mathrm{H}+\mathrm{D} \rightarrow \mathrm{HD}$ & \\
\hline 18 & $\mathrm{D}+\mathrm{D} \rightarrow \mathrm{D}_{2}$ & \\
\hline 19 & $\mathrm{D}+\mathrm{O} \rightarrow \mathrm{OD}$ & \\
\hline 20 & $\mathrm{D}+\mathrm{OH} \rightarrow \mathrm{HDO}$ & \\
\hline 21 & $\mathrm{H}+\mathrm{OD} \rightarrow \mathrm{HDO}$ & \\
\hline 22 & $\mathrm{D}+\mathrm{OD} \rightarrow \mathrm{D}_{2} \mathrm{O}$ & \\
\hline 23 & $\mathrm{D}+\mathrm{CO} \rightarrow \mathrm{DCO}$ & 320 \\
\hline 24 & $\mathrm{H}+\mathrm{DCO} \rightarrow \mathrm{HDCO}$ & \\
\hline 25 & $\mathrm{D}+\mathrm{HCO} \rightarrow \mathrm{HDCO}$ & \\
\hline 26 & $\mathrm{D}+\mathrm{H}_{2} \mathrm{CO} \rightarrow \mathrm{H}_{2} \mathrm{DCO}$ & 214 \\
\hline 27 & $\mathrm{D}+\mathrm{HDCO} \rightarrow \mathrm{HD}_{2} \mathrm{CO}$ & 173 \\
\hline 28 & $\mathrm{D}+\mathrm{D}_{2} \mathrm{CO} \rightarrow \mathrm{D}_{3} \mathrm{CO}$ & 128 \\
\hline 29 & $\mathrm{H}+\mathrm{HDCO} \rightarrow \mathrm{H}_{2} \mathrm{DCO}$ & 380 \\
\hline 30 & $\mathrm{H}+\mathrm{D}_{2} \mathrm{CO} \rightarrow \mathrm{HD}_{2} \mathrm{CO}$ & 340 \\
\hline 31 & $\mathrm{D}+\mathrm{H}_{3} \mathrm{CO} \rightarrow \mathrm{CH}_{3} \mathrm{OD}$ & \\
\hline 32 & $\mathrm{D}+\mathrm{H}_{2} \mathrm{DCO} \rightarrow \mathrm{CH}_{2} \mathrm{DOD}$ & \\
\hline 33 & $\mathrm{D}+\mathrm{HD}_{2} \mathrm{CO} \rightarrow \mathrm{CHD}_{2} \mathrm{OD}$ & \\
\hline 34 & $\mathrm{D}+\mathrm{D}_{3} \mathrm{CO} \rightarrow \mathrm{CD}_{3} \mathrm{OD}$ & \\
\hline 35 & $\mathrm{H}+\mathrm{H}_{2} \mathrm{DCO} \rightarrow \mathrm{CH}_{3} \mathrm{OD}$ & \\
\hline 36 & $\mathrm{H}+\mathrm{HD}_{2} \mathrm{CO} \rightarrow \mathrm{CH}_{2} \mathrm{DOD}$ & \\
\hline 37 & $\mathrm{H}+\mathrm{D}_{3} \mathrm{CO} \rightarrow \mathrm{CHD}_{2} \mathrm{OD}$ & \\
\hline 38 & $\mathrm{O}+\mathrm{DCO} \rightarrow \mathrm{CO}_{2}+\mathrm{D}$ & \\
\hline 39 & $\mathrm{D}+\mathrm{O}_{2} \rightarrow \mathrm{DO}_{2}$ & \\
\hline 40 & $\mathrm{D}+\mathrm{HO}_{2} \rightarrow \mathrm{HDO}_{2}$ & \\
\hline 41 & $\mathrm{D}+\mathrm{DO}_{2} \rightarrow \mathrm{D}_{2} \mathrm{O}_{2}$ & \\
\hline 42 & $\mathrm{H}+\mathrm{DO}_{2} \rightarrow \mathrm{HDO}_{2}$ & \\
\hline 43 & $\mathrm{D}+\mathrm{O}_{3} \rightarrow \mathrm{O}_{2}+\mathrm{OD}$ & 450 \\
\hline 44 & $\mathrm{H}_{2}+\mathrm{OD} \rightarrow \mathrm{HDO}+\mathrm{H}$ & 2600 \\
\hline 45 & $\mathrm{HD}+\mathrm{OD} \rightarrow \mathrm{HDO}+\mathrm{D}$ & 2600 \\
\hline 46 & $\mathrm{HD}+\mathrm{OD} \rightarrow \mathrm{D}_{2} \mathrm{O}+\mathrm{H}$ & 2600 \\
\hline 47 & $\mathrm{HD}+\mathrm{OH} \rightarrow \mathrm{H}_{2} \mathrm{O}+\mathrm{D}$ & 2600 \\
\hline 48 & $\mathrm{D}_{2}+\mathrm{OD} \rightarrow \mathrm{D}_{2} \mathrm{O}+\mathrm{D}$ & 2600 \\
\hline P1 & $\mathrm{O}_{2} \rightarrow \mathrm{O}+\mathrm{O}$ & \\
\hline P2 & $\mathrm{OH} \rightarrow \mathrm{O}+\mathrm{H}$ & \\
\hline P3 & $\mathrm{H}_{2} \mathrm{O} \rightarrow \mathrm{H}+\mathrm{OH}$ & \\
\hline $\mathrm{P} 4$ & $\mathrm{CO} \rightarrow \mathrm{C}+\mathrm{O}$ & \\
\hline P5 & $\mathrm{HCO} \rightarrow \mathrm{H}+\mathrm{CO}$ & \\
\hline P6 & $\mathrm{H}_{2} \mathrm{CO} \rightarrow \mathrm{H}_{2}+\mathrm{CO}$ & \\
\hline P7 & $\mathrm{CH}_{3} \mathrm{OH} \rightarrow \mathrm{CH}_{3}+\mathrm{OH}$ & \\
\hline P8 & $\mathrm{CO}_{2} \rightarrow \mathrm{O}+\mathrm{CO}$ & \\
\hline P9 & $\mathrm{H}_{2} \mathrm{O}_{2} \rightarrow \mathrm{OH}+\mathrm{OH}$ & \\
\hline P10 & $\mathrm{O}_{3} \rightarrow \mathrm{O}_{2}+\mathrm{O}$ & \\
\hline P12 & $\mathrm{HO}_{2} \rightarrow \mathrm{OH}+\mathrm{O}$ & \\
\hline $\mathrm{P} 13$ & $\mathrm{OD} \rightarrow \mathrm{O}+\mathrm{D}$ & \\
\hline P14 & $\mathrm{D}_{2} \mathrm{O} \rightarrow \mathrm{D}+\mathrm{OD}$ & \\
\hline P15 & $\mathrm{HDO} \rightarrow \mathrm{H}+\mathrm{OD}$ & \\
\hline P16 & $\mathrm{DCO} \rightarrow \mathrm{D}+\mathrm{CO}$ & \\
\hline P17 & $\mathrm{HDCO} \rightarrow \mathrm{HD}+\mathrm{CO}$ & \\
\hline P18 & $\mathrm{D}_{2} \mathrm{CO} \rightarrow \mathrm{D}_{2}+\mathrm{CO}$ & \\
\hline P19 & $\mathrm{CH}_{3} \mathrm{OD} \rightarrow \mathrm{CH}_{3}+\mathrm{OD}$ & \\
\hline P20 & $\mathrm{CH}_{2} \mathrm{DOH} \rightarrow \mathrm{CH}_{2} \mathrm{D}+\mathrm{OH}$ & \\
\hline P21 & $\mathrm{CHD}_{2} \mathrm{OH} \rightarrow \mathrm{CHD}_{2}+\mathrm{OH}$ & \\
\hline $\mathrm{P} 22$ & $\mathrm{CH}_{2} \mathrm{DOD} \rightarrow \mathrm{CH}_{2} \mathrm{D}+\mathrm{OD}$ & \\
\hline $\mathrm{P} 23$ & $\mathrm{CD}_{3} \mathrm{OH} \rightarrow \mathrm{CD}_{3}+\mathrm{OH}$ & \\
\hline P24 & $\mathrm{CHD}_{2} \mathrm{OD} \rightarrow \mathrm{CHD}_{2}+\mathrm{OD}$ & \\
\hline P25 & $\mathrm{CD}_{3} \mathrm{OD} \rightarrow \mathrm{CD}_{3}+\mathrm{OD}$ & \\
\hline P26 & $\mathrm{HDO}_{2} \rightarrow \mathrm{OD}+\mathrm{OH}$ & \\
\hline P27 & $\mathrm{D}_{2} \mathrm{O}_{2} \rightarrow \mathrm{OD}+\mathrm{OD}$ & \\
\hline P28 & $\mathrm{DO}_{2} \rightarrow \mathrm{OD}+\mathrm{O}$ & \\
\hline
\end{tabular}


Ankan Das, Dipen Sahu, Liton Majumdar, Sandip K. Chakrabarti

(having $\sim 10^{6}$ sites). Reason for choosing $50 \times 50$ site grains is that it is well above the limit of the statistical fluctuation (Chang. Cuppen \& Herbst 2005). Here, all the events are processed by generating random numbers. Both types of reaction mechanisms, namely, Langmuir-Hinshelwood (reactions between surface species via hopping) and Eley-Rideal (reaction between incoming species with surface species) are considered.

We assume that only gas phase $\mathrm{H}, \mathrm{D}, \mathrm{O}$ and $\mathrm{CO}$ are accreting on the grain surface. Unless otherwise stated, sticking probability $\left(s_{n}\right)$ of all accreting species is assumed to be 1 . Initial gas phase number densities of these species are taken from the parameter space proposed by Das. Acharvva \& Chakrabarti (2010). Initial abundances of these species for $n_{H}=10^{4} \mathrm{~cm}^{-3}$ are presented in Table 1. For the other density regions, initial abundances of these species are scaled accordingly. Initial abundance of gas phase atomic deuterium is assumed to be $r_{d}$ times the abundance of gas phase atomic hydrogen, where, $r_{d}$ is the initial atomic $\mathrm{D} / \mathrm{H}$ ratio. Unless otherwise mentioned, for most of our calculations, we use $r_{d}=0.3$ by following Caselli (2002); Osamura, Roberts \& Herbst (2004). Since here we consider gas-grain interaction over the time, gas phase abundances of $\mathrm{O}$ and $\mathrm{CO}$ are considered to be depleted.

The reaction network considered here is shown in Table 2. Activation barriers are used by following Das \& Chakrabarti (2011) and references therein. These barriers are also shown in Table 2. For the deuterated reactions, activation barriers could differ due to zero point vibrations (Das et al. 2015b; Caselli 2002). We follow Caselli (2002) and updated activation barriers presented in Fuchs et al. (2009) to update our activation barriers for the deuterated reactions. As in Das \& Chakrabarti (2011), here also, we consider some photo-reactions with similar rate constants. Photo-reactions for the deuterated analogues are considered to have similar rate constants.

Binding energy of surface species controls the chemical composition of the interstellar grain mantle. Normally, surface binding energies are computed from various theoretical calculations (such as: Allen \& Robinson (1977)). From experimental findings of Pirronello et al. (1997); Pirronello et al. (1999), it was interpreted by Katz et al. (1999) that atomic hydrogen moves slower than what is usually assumed in various simulations. Here, these experimental findings are also incorporated. Since D atoms are heavier than $\mathrm{H}$ atoms, Caselli (2002) used $2 \mathrm{meV}$ higher diffusion energy for D atom than $\mathrm{H}$ atom. Following this assumption, Lipshtat. Biham \& Herbst (2004) considered a $2 \mathrm{meV}$ difference between the diffusion energies of $\mathrm{H}$ and $\mathrm{D}$ atom. Lipshtat, Biham \& Herbst (2004) also considered a $10 \mathrm{meV}$ energy difference between the desorption energies of $\mathrm{H}$ and D atoms. In Table 3, we show 4 Sets of binding energies which are considered in our simulation. Since for all other species, we consider binding energies to be the same as in Das, Acharvva \& Chakrabarti (2010); Das \& Chakrabarti (2011), only binding energies of $\mathrm{H}, \mathrm{D}, \mathrm{H}_{2}, \mathrm{HD}$ and $\mathrm{D}_{2}$ are shown in the Table 3. Set 1 corresponds to the theoretical values obtained by Allen \& Robinson (1977). Unless otherwise stated, we always use Set 1 energy values. In Set 2 and Set 3, experimental findings (Katz et al. 1999) of energies for olivine and amorphous carbon grains are respectively shown. Except $\mathrm{H}, \mathrm{D}, \mathrm{H}_{2}, \mathrm{HD}, \mathrm{D}_{2}$, all other energy values were kept unchanged as in Set 1. Due to the unavailability of energy barriers, for Set 1 , Set 2 and Set 3, binding energies of $\mathrm{HD}$ and $\mathrm{D}_{2}$ are assumed to be similar to $\mathrm{H}_{2}$. In Set 4 , considerations of Lipshtat. Biham \& Herbst (2004) are taken care of. Since, no energy barriers for $\mathrm{H}_{2}, \mathrm{HD}$ and $\mathrm{D}_{2}$ were available, we consider $E_{D}\left(\mathrm{H}_{2}\right) / E_{D}(\mathrm{H})$ ratios of energy values of Sets 2 and 3 for the computation of $E_{D}\left(\mathrm{H}_{2}\right)$ from $E_{D}(\mathrm{H})$ of Lipshtat, Biham \& Herbst (2004) case. A similar ratio $\left(E_{D}\left(\mathrm{H}_{2}\right) / E_{D}(\mathrm{H})\right)$ is also used to compute $E_{D}\left(\mathrm{HD}, \mathrm{D}_{2}\right)$ from $E_{D}(\mathrm{D})$ of Lipshtat. Biham \& Herbst (2004) case.

\subsection{Modeling the Physical Aspects}

The chemical composition of interstellar grain mantle solely depends on the surrounding physical conditions and age of the cloud. Presently, we wish to study chemical composition of interstellar grain mantles for various types of clouds. Snow \& McCall (2006) classified clouds depending on densities $\left(n_{H}\right)$, visual extinctions $\left(A_{V}\right)$ and temperatures $(T)$. Here also, we are considering similar classification for our simulations. For the diffuse cloud region, we consider that $n_{H}$ may vary between $10^{2}-5.00 \times 10^{2} \mathrm{~cm}^{-3}, T$ may vary in between $30-100 \mathrm{~K}$ and $A_{V}=0.2$, for the translucent cloud region, we use $n_{H}=5.01 \times 10^{2}-10^{4} \mathrm{~cm}^{-3}, T=15-30$ $\mathrm{K}, A_{V}=1-2$ and for the dense cloud region, we use $n_{H}=1.0001 \times 10^{4}-10^{6} \mathrm{~cm}^{-3}, T=10 \mathrm{~K}$ and $A_{V}=5-10$. In order to consider more realistic condition, we consider different temperatures and extinction parameters for various regions of clouds. We use constant slopes for $T$ and $A_{V}$ in respective number density windows. Fig. 1 clearly shows our choice of physical parameters. In diffuse and translucent clouds regions, gas and grains are not well coupled. So, temperature between these two phases might vary. However, in dense cloud regions gas and grains are strongly coupled and the temperature would be more or less the same for both the phases. Here, we assume that the grain temperature $\left(T_{g r}\right)$ is always fixed at $10 \mathrm{~K}$ for all the clouds and gas temperature $\left(T_{\text {gas }}\right)$, we assume $T_{\text {gas }}=T$.

\section{RESULTS}

\subsection{Composition of grain mantles with deuterated species}

Despite low elemental abundance of atomic deuterium in interstellar space, several species are found to be heavily fractionated in that sense, hydrogen atom is replaced by deuterium. In order to find out the effects of this trace amount of deuterium on the chemical composition, we study both the cases: without (Fig. 2a) and with deuterium (Fig. 2b, Fig. 2c, Fig. 2d and Fig. 2e). Here, we consider $n_{H}=2 \times 10^{4}$ $\mathrm{cm}^{-3}$ and corresponding values of $T_{\text {gas }}$ and $A_{V}$ are chosen according to Fig. 1. For Fig. 2a, 2b, Fig. 2c and Fig. 2d, we use $r_{d}=0,0.01,0.1$ and 0.3 respectively and $s_{n}=1$. In case of Fig. 2e, we use $r_{d}=0.3$ but consider $s_{n}=0.3$ to see the effect of sticking parameter on the composition of grain mantle. The difference between Fig. $2 d$ and Fig. 2e is clear. Consideration of the lower sticking probability largely affects the mantle composition. Formation of numbers of layers is significantly lower in case of Fig. 2e. Fig. 2b, Fig 2c and Fig. 
Deuterium enrichment of the interstellar grain mantle

Table 3. Different sets of binding energies

\begin{tabular}{|c|c|c|c|c|c|c|c|c|}
\hline \multirow[t]{2}{*}{ Species } & \multicolumn{2}{|c|}{$\begin{array}{c}\text { Set } 1 \\
\text { (Allen \& Robinson 1977) } \\
\end{array}$} & \multicolumn{2}{|c|}{$\begin{array}{c}\text { Set } 2 \\
\text { (Katz et al. (1999) for olivine) }\end{array}$} & \multicolumn{2}{|c|}{$\begin{array}{c}\text { Set } 3 \\
\text { (Katz et al. (1999) for amorphous) }\end{array}$} & \multicolumn{2}{|c|}{$\begin{array}{c}\text { Set } 4 \\
\text { (Lipshtat, Biham \& Herbst 2004) } \\
\end{array}$} \\
\hline & $\mathbf{E}_{\mathbf{b}}(\mathbf{K})$ & $\mathbf{E}_{\mathbf{d}}(\mathbf{K})$ & $\mathbf{E}_{\mathbf{b}}(\mathbf{K})$ & $\mathbf{E}_{\mathbf{d}}(\mathbf{K})$ & $\mathbf{E}_{\mathbf{b}}(\mathbf{K})$ & $\mathbf{E}_{\mathbf{d}}(\mathbf{K})$ & $\mathbf{E}_{\mathbf{b}}(\mathbf{K})$ & $\mathbf{E}_{\mathbf{d}}(\mathbf{K})$ \\
\hline $\mathbf{H}$ & 100 & 350 & 287 & 373 & 511 & 657 & 406 & 580 \\
\hline D & 100 & 350 & 287 & 373 & 511 & 657 & 429 & 697 \\
\hline $\mathbf{H}_{2}$ & 135 & 450 & 94 & 314 & 163 & 542 & 143 & 478 \\
\hline HD & 135 & 450 & 94 & 314 & 163 & 542 & 173 & 575 \\
\hline $\mathbf{D}_{2}$ & 135 & 450 & 94 & 314 & 163 & 542 & 173 & 575 \\
\hline
\end{tabular}

$2 \mathrm{~d}$ show the progressive increment of deuterium fractionation in comparison to the non-deuterated case (Fig. 2a). For a better assessment, surface coverage, deuterium fractionation and abundances of some important surface species are shown in Table 4 . Table 4 gives a comprehensive knowledge about various aspects of deuterium enrichment shown in Fig. 2(a-e). For simplicity, we are discussing only the case of $r_{d}=0.3$ and $s_{n}=1$ here.

From Fig. 2a $\left(r_{d}=0\right)$, we find that water and methanol are maintaining surface coverages of $85.5 \%$ and $7.8 \%$ respectively. In case of Fig. $2 \mathrm{~d}$, where $r_{d}=0.3$ is considered, surface coverage of water in all forms and methanol in all forms come out to be $86 \%$ (normal water is $53.6 \%$ and deuterated water is $32.4 \%$ ) and $8.5 \%$ (normal methanol is $4.6 \%$ and deuterated methanol is $2.9 \%$ ) respectively. Among the deuterated species, HDO is mostly dominating (surface coverage $29 \%$ ) while $\mathrm{D}_{2} \mathrm{O}$ is also found to be abundant (surface coverage $3.4 \%$ ). Deuterium fractionation of singly deuterated methanols, i.e., for $\mathrm{CH}_{3} \mathrm{OD}$ and $\mathrm{CH}_{2} \mathrm{DOH}$ are found to be 0.27 and 0.28 respectively and seems to occupy significant percentage of the grain mantle $(1.25 \%$ and $1.27 \%$ respectively). For a similar choice of $r_{d}$, fractionation values for the doubly deuterated methanols are found to be 0.110 and 0.105 respectively for $\mathrm{CHD}_{2} \mathrm{OH}$ and $\mathrm{CH}_{2}$ DOD. For a similar situation, triply deuterated methanols possess fractionation ratios of 0.04 and 0.03 respectively for $\mathrm{CD}_{3} \mathrm{OH}$ and $\mathrm{CHD}_{2} \mathrm{OD}$. For $\mathrm{CD}_{3} \mathrm{OD}$, this ratio is found to be 0.008 .

Triply deuterated methanol $\left(\mathrm{CD}_{3} \mathrm{OH}\right)$ has recently been observed to have a fractionation ratio of $0.014( \pm 0.014) / 0.008( \pm 0.006))$ in IRAS 162932422 by Parise et al. (2004). IRAS 16293-2422 is a Class 0 protostellar binary. It is expected that due to the heating of newly formed stars, ice mantles are evaporated and grain phase species are released into the gas phase (charnley. Tielens \& Millar 1992; Charnlev. Tielens \& Rodgers 1997; Caselli 1993; Parise et al. 2004). One would expect that deuterium fractionation of the ice phase would be preserved in the gas phase as well but this is not true for all the species. In Table 4, we compare our calculated water and methanol abundance/fractionation with existing observational results. Doubly deuterated methanol $\left(\mathrm{CHD}_{2} \mathrm{OH}\right)$ is also observed by Parise et al. (2004) having a fractionation ratio of $0.06( \pm 0.05)$. Our calculated results for $r_{d}=0.3$ is in good agreement for the fractionation ratios for doubly and triply deuterated methanol. In case of singly deuterated methanols, we are having roughly similar fractionation ratios. Our obtained result for $\mathrm{CH}_{2} \mathrm{DOH}$ is very close to the observed value. But observational evidences suggest that $\mathrm{CH}_{3} \mathrm{OD}$ is relatively less abundant in comparison to $\mathrm{CH}_{2} \mathrm{DOH}$ and fractionation ratio of $\mathrm{CH}_{3} \mathrm{OD} / \mathrm{CH}_{3} \mathrm{OH}$ and $\mathrm{CH}_{2} \mathrm{DOH} / \mathrm{CH}_{3} \mathrm{OH}$ would be $0.02( \pm 0.01)$ and $0.3( \pm 0.2)$ respectively in IRAS 16293-2422. Charnley. Tielens \& Rodgers (1997) and Osamura, Roberts \& Herbst (2004) discussed the possible interconversion between pairs of deuterated forms of methanol, its ion, and its proportionated ion in star-forming regions. They also suggested that $\mathrm{CH}_{3} \mathrm{OD}$ is comparatively less abundant than $\mathrm{CH}_{2} \mathrm{DOH}$. Osamura, Roberts \& Herbst (2004) carried out quantum chemical calculations to show various energy required for the possible internal rearrangements. They assumed that methanol along with its deuterated forms mainly form on interstellar dust and are evaporated into gas phase by several other means and the interconversion mainly take place after the evaporation from the dusts. They ran some protostellar models, starting immediately after the loss of grain mantles. Here, we consider only depletions of some gas phase species. Evaporation of surface species is considered by cosmic ray induced process and thermal process only. Since we consider grain temperature to be fixed at $10 \mathrm{~K}$, in our case, no such bulk evaporation takes place. Consideration of detailed gas phase chemistry along with the Monte Carlo simulation is also out of scope for this paper. Thus the fractionation ratios obtained from our simulation only reflects the values in the cold phase only. This ratio might be changed after the evaporation of grain mantles because of the possible interaction with some dominant gas phase species. Caselli (2002) also studied the formation of deuterated isotopomers of methanol on interstellar grain surfaces with a semi-empirical modified rate approach and a Monte Carlo method in between the temperature range of $10-20 \mathrm{~K}$. For intermediate density cloud $\left(n_{H}=10^{4} \mathrm{~cm}^{-3}\right)$, their Monte Carlo simulation showed that the fractionation ratios after $10^{4}$ year with an initial atomic $\mathrm{D} / \mathrm{H}$ ratio of 0.3 are $0.19,0.19,3.3 \times 10^{-4}, 0.034$ and $6.6 \times 10^{-5}$ respectively for $\mathrm{CH}_{3} \mathrm{OD}, \mathrm{CH}_{2} \mathrm{DOH}, \mathrm{CHD}_{2} \mathrm{OH}, \mathrm{CHD}_{2} \mathrm{OD}$ and $\mathrm{CHD}_{2} \mathrm{OD}$. Since they also did not follow the time evolution of the gas phase species after the bulk evaporation, they also obtained similar fractionation ratios for the singly deuterated methanols.

Though a high level of deuterium fractionation of methanol is evident from the observations, surprisingly, a low fractionation ratio is observed for HDO. This is in contradiction with our results. Origin of this discrepancy is yet to be understood and could indicate new processes not incorporated in our study. Persson, Jørgensen, van Dishoeck (2012) measured deuterium fractionation in the warm gas of the deeply-embedded protostellar binary IRAS 16293-2422. They found a fractionation ratio of $9.2 \pm 2.6 \times 10^{-4}$ for 
Table 4. Surface coverage, deuterium fractionation and abundances of various surface species with the absence and presence of deuterium.

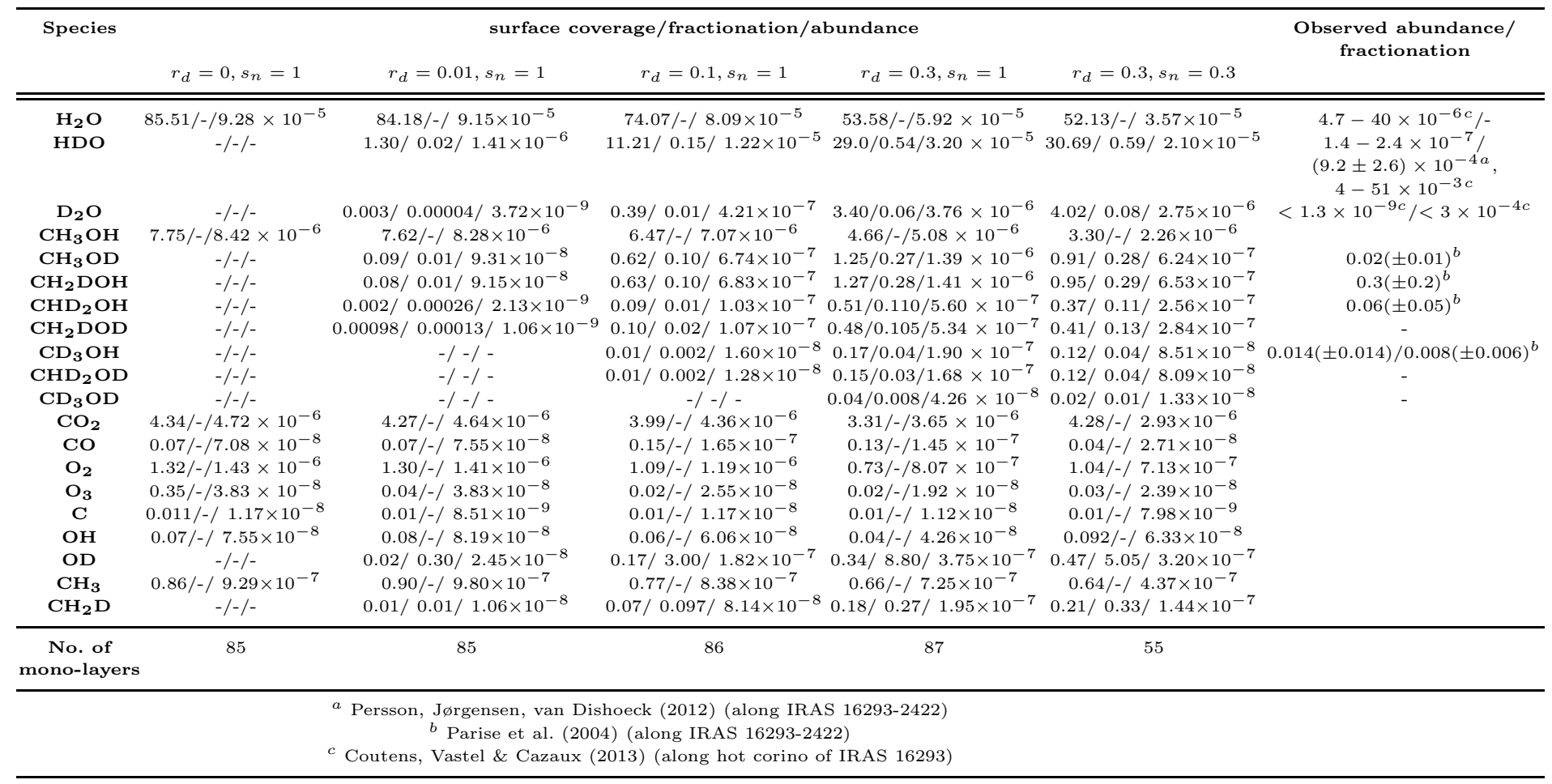

solid HDO. Persson, Jørgensen, van Dishoeck (2012) predicted an upper limit of $10^{-2}$ for $\mathrm{HDO} / \mathrm{H}_{2} \mathrm{O}$ in NGC 7358 IRS9. Parise et al. (2003) discusses various scenarios which could explain such low water deuteration compared to the observed high deuteration of methanol in the gas-phase. Wakelam et al. (2014) model the chemistry occurring on IRAS 16293-2422 with a large gas-grain chemical code. They used various physical conditions to reproduce the observed $\mathrm{HDO} / \mathrm{H}_{2} \mathrm{O}$ and $\mathrm{D}_{2} \mathrm{O} / \mathrm{H}_{2} \mathrm{O}$. They found that by considering a cosmic ray ionization rate of $10^{-16} \mathrm{~S}^{-1}$ and $\mathrm{C} / \mathrm{O}$ elemental ratio of 0.5 , observed ratios would be reproduced around the central part of the protostellar envelope but their model was not able to reproduce the observed ratios for the outer part of the protostellar envelope. This important unresolved puzzle clearly requires further investigation.

\subsection{Time evolution of mantle composition}

Results presented in Fig. 2(a-e) and Table 4 represent the status after $2 \times 10^{6}$ years. We call this time as the 'standard time'. Fig. 2(a-e) shows that several mono-layers would be produced during the simulation period. Studies by Flower et al. (2006) suggested that evolution of layers can take place even in longer time scales. In order to see the effects of time on the formation of number of mono-layers, we carry out our simulation for much longer time scales ( $10^{7}$ years). Fig. 3 shows the formation of number of layers in different time scales. 'Early time' corresponds to $10^{4}$ years, 'intermediate time' corresponds to $10^{5}$ years and 'late time' corresponds to $10^{7}$ years. By the number of layers, we mean the maximum number of layers counting from the bare grain surface on at least one site. Thus, $n^{\text {th }}$ layer starts with the first instance of deposition of any species on any site which already has (n-1) layer. From Fig. 3, it is clear that the formation of number of layers is highly time dependent phenomenon. For the low density region, formation of number of layers is linearly increasing with time. Situation is somewhat different in the high density region. For the high density region, i.e., when $n_{H}>2 \times 10^{4} \mathrm{~cm}^{-3}$ region, hardly a few new layers are produced beyond standard time. Thus it is not useful to continue simulation for longer period. Thus to save computational time, we computed only up to the standard time and showed our results.

This aspect would be better understood from Fig. 4(ac), where chemical evolution of the most dominating gasgrain species of our chemical network is shown for $n_{H}=10^{3}$, $2 \times 10^{4} \& 10^{6} \mathrm{~cm}^{-3}$ cloud. Corresponding values of the temperatures and extinction parameters are chosen from Fig. 1. Along the 'y' axis of Fig. 4(a-c), we show the abundances of gas-grain species with respect to total hydrogen nuclei in all form and ' $\mathrm{x}$ ' axis shows time in years. For this case, we continue our simulation up to $10^{7}$ years. For the low density region $\left(n_{H}=10^{3} \mathrm{~cm}^{-3}\right)$, steady state between the surface species is not achieved. For the higher density regions $\left(n_{H}=2 \times 10^{4}\right.$ and $\left.10^{6} \mathrm{~cm}^{-3}\right)$, a steady state among the surface species would arise within the standard time. This is possible due to depletion of gas phase species.

For Fig. 4a (low density), we use $n_{H}=10^{3} \mathrm{~cm}^{-3}$ and strong radiation field $\left(A_{V} \sim 1.05\right)$. In this low density region, formation of complex species is mainly countered by 
the photo-dissociation process. Due to this reason, photo dissociation products like $\mathrm{C}, \mathrm{CH}_{3}, \mathrm{CH}_{2} \mathrm{D}, \mathrm{OH}, \mathrm{OD}$ are covering significant parts of the grain mantles. For the high density regions, gas phase $\mathrm{O}$ and $\mathrm{CO}$ are heavily depleted and steady state is achieved in much shorter time scales. Both the deuterated forms of $\mathrm{H}_{2} \mathrm{O}$ (HDO \& $\mathrm{D}_{2} \mathrm{O}$ ) are produced efficiently when $n_{H}=2 \times 10^{4} \mathrm{~cm}^{-3}$. Singly deuterated forms of methanol $\left(\mathrm{CH}_{3} \mathrm{OD}\right.$ and $\left.\mathrm{CH}_{2} \mathrm{DOH}\right)$ are also found to be covering a significant percentage of grain mantles. $\mathrm{O}_{3}$ is found to be the most dominating species in the much higher density region $\left(10^{6}\right)$. Fig. 4(a-c) clearly shows how time affects the abundances of gas-grain species for the low density region. However, for the sake of benchmarking, we need to choose a time scale to compare various aspects of grain mantles.

\subsection{Analysis of the grain mantle composition in different types of clouds}

In Fig. 5(a-e), we show the composition of grain mantles for various cloud regions. To represent diffuse cloud region, we use $n_{H}=10^{2} \mathrm{~cm}^{-3}$ (Fig. 5a). For a translucent cloud, we use $n_{H}=10^{3} \mathrm{~cm}^{-3}$ (Fig. 5b) and $n_{H}=5 \times 10^{3} \mathrm{~cm}^{-3}$ (Fig. $5 \mathrm{c})$. To represent a dense cloud region, we use $n_{H}=2 \times 10^{4}$ $\mathrm{cm}^{-3}$ (Fig. 5d) and $n_{H}=10^{6} \mathrm{~cm}^{-3}$ (Fig. 5e). Respective values of $T_{g a s}, T_{g r}, A_{V}$ are chosen according to the physical profiles described in Fig. 1. Mantle compositions are plotted after a standard time. Surface coverage, fractionation and abundances of some important surface species are given in Table 5.

In diffuse cloud regions (Fig. 5a), the radiation field is very strong. So hardly a few layers would be produced. In translucent cloud regions (Fig. 5b and Fig. 5c), the radiation field is somewhat attenuated but its effect is till there. Thus the surface coverage is mainly dominated by the photo-dissociation products such as $\mathrm{C}, \mathrm{CH}_{3}, \mathrm{CH}_{2} \mathrm{D}, \mathrm{OH}, \mathrm{OD}$ etc. Formation of number of layers is progressively higher for when the density is increased from $10^{3} \mathrm{~cm}^{-3}$ to $5 \times 10^{3}$ $\mathrm{cm}^{-3}$. For a higher density region (Fig. $5 \mathrm{~d}$ and Fig. 5e), radiation field is almost attenuated. Thus, formation of complex molecules are favoured. One interesting fact could be noted from Table 5 that as we are going to the higher density side of the dense cloud region (from $n_{H}=2 \times 10^{4} \mathrm{~cm}^{-3}$ to $10^{6}$ $\mathrm{cm}^{-3}$ ), surface coverage of $\mathrm{H}_{2} \mathrm{O}$ and $\mathrm{CH}_{3} \mathrm{OH}$ along with its deuterated forms drastically drops. In general, abundances of methanol and water increases with density and attain a maximum around $n_{H}=2 \times 10^{4} \mathrm{~cm}^{-3}$. If we further increase the density beyond $n_{H}=2 \times 10^{4} \mathrm{~cm}^{-3}$, their abundance starts to drop. So there are actually two regions, one region is extended from $10^{2} \mathrm{~cm}^{-3}$ to $2 \times 10^{4} \mathrm{~cm}^{-3}$ and another is beyond $n_{H}=2 \times 10^{4} \mathrm{~cm}^{-3}$. From Table 5 it is evident that water abundance which was $2.48 \times 10^{-7}$ in $n_{H}=10^{2}$ $\mathrm{cm}^{-3}$ region, increases to $5.92 \times 10^{-5}$ in $n_{H}=2 \times 10^{4} \mathrm{~cm}^{-3}$ region. Beyond $n_{H}=2 \times 10^{4} \mathrm{~cm}^{-3}$, it starts to drop and at $n_{H}=10^{6} \mathrm{~cm}^{-3}$ it comes out to be $6.55 \times 10^{-6}$. Similar is also true for methanol. Production of methanol for the diffuse cloud region $\left(n_{H}=10^{2} \mathrm{~cm}^{-3}\right)$ was not significant but its production steadily increase with the increase in density and its abundance comes out to be $5.08 \times 10^{6}$ in $n_{H}=2 \times 10^{4} \mathrm{~cm}^{-3}$. Beyond $2 \times 10^{4} \mathrm{~cm}^{-3}$, methanol abundance starts to decrease and finally comes out to be $5.15 \times 10^{-7}$ for $n_{H}=10^{6} \mathrm{~cm}^{-3}$. On the contrary, around the second region $\left(n_{H}=2 \times 10^{4} \mathrm{~cm}^{-3}\right.$ to $\left.10^{6} \mathrm{~cm}^{-3}\right)$, surface coverage of $\mathrm{CO}, \mathrm{CO} 2, \mathrm{O}_{2}, \mathrm{O}_{3}$ significantly increases. Since for $2 \times 10^{4} \mathrm{~cm}^{-3}$ accretion rate is comparatively lower, surface species gets adequate time to recombine and produce various hydrogenated or deuterated species. This region of cloud is found to be favourable for the production of maximum numbers of species and thus forming maximum number of layers. Fig. 3 also shows that beyond the standard time, the region with $n_{H}=2 \times 10^{4} \mathrm{~cm}^{-3}$ produces maximum number of layers. In this region, most of the incoming $\mathrm{H}$ and $\mathrm{D}$ atoms are utilized to form new molecules because they get adequate time for the reactions. Thus this region produces more numbers of molecules and more numbers of layers. For the higher density side, accretion rate is high and $\mathrm{H}$ and $\mathrm{D}$ would be blocked by any unwanted species next to them. Thus $\mathrm{H}$ and $\mathrm{D}$ would evaporate from that site without making any reaction. Thus less number of species would be produced and less number of layers would be produced when $n_{H}>2 \times 10^{4} \mathrm{~cm}^{-3}$. For the low density region, since the accretion rate is comparatively lower, it is not expected to produce more layers within the specified time. As we are increasing the density (diffuse to dense), Fig. 3 shows an increasing profile for the standard time. However, for $n_{H}>2 \times 10^{4} \mathrm{~cm}^{-3}$, numbers of layers are decreasing. For example, in case of $n_{H}=10^{6} \mathrm{~cm}^{-3}$ region, comparatively less numbers of layers are produced. Here, the surface is full of various species, so $\mathrm{H}$ or $\mathrm{D}$ atoms could be easily blocked by other unfavourable species (reactions either not favourable or possess high activation barrier) and are thermally evaporated after their residence time. Thus formation of hydrogenated or deuterated species are heavily hindered. As a result, CO mostly remain unutilized on the grain. Atomic oxygens, which are mostly converted to $\mathrm{H}_{2} \mathrm{O}$ and its deuterated forms in case of $n_{H}=2 \times 10^{4} \mathrm{~cm}^{-3}$ region, remain unutilized here and channelized to form $\mathrm{O}_{2}, \mathrm{O}_{3}$ and $\mathrm{CO}_{2}$. For better illustration purpose, in Fig. 6, we show abundances of various surface species in relation to number density. Fig. 6 clearly shows that water and methanol abundances steadily increase with the increase in $n_{H}$. However, when $n_{H}>2 \times 10^{4} \mathrm{~cm}^{-3}$, they are significantly decreased. Throughout the regions, abundances of $C O$ is found to be linearly increasing with the number density of clouds. Abundance of $\mathrm{CO}_{2}$ is found to increase with the increase in $n_{H}$ but beyond $n_{H}=2 \times 10^{4} \mathrm{~cm}^{-3}$, it roughly maintains a steady state. Photo-dissociation products which are abundant around the diffuse and translucent cloud regions are sharply decreasing in regions with $n_{H}>5 \times 10^{3} \mathrm{~cm}^{-3}$.

\subsection{Composition of grain mantles for various sets of binding energies}

Till now, we used Set 1 energy barriers. Presently, we consider all 4 sets of energy barriers given in Table 3. Chemical composition of the grain mantles under these energy barriers is shown in Fig. $7(\mathrm{a}-\mathrm{d})$. In this case, we use $n_{H}=2 \times 10^{4}$ $\mathrm{cm}^{-3}$ and choose $A_{V}, T_{g a s}, T_{g r}$ from Fig. 1. For quantitative comparison, we show the surface coverage, deuterium fractionation and abundances of important surface species in Table 6 . It is clear from Fig. $7(\mathrm{a}-\mathrm{d})$ and Table 6 that methanol is efficiently produced for Set 1 binding energies. Hydrogenation reactions are the fastest reactions on the grain surface. Since for Set 1, energy barrier against diffusion is the low- 


\section{Ankan Das, Dipen Sahu, Liton Majumdar, Sandip K. Chakrabarti}

Table 5. Surface coverage, fractionation ratio and abundance of some important surface species for various types of clouds.

\begin{tabular}{|c|c|c|c|c|c|}
\hline \multirow[t]{3}{*}{ Species } & \multicolumn{5}{|c|}{ Surface coverage/deuterium fractionation/ abundance } \\
\hline & \multicolumn{2}{|c|}{ Dense cloud } & \multicolumn{2}{|c|}{ Translucent cloud } & \multirow{2}{*}{$\begin{array}{l}\text { Diffuse cloud } \\
10^{2} \mathbf{c m}^{-3}\end{array}$} \\
\hline & $n_{H}=10^{6} \mathbf{c m}^{-3}$ & $n_{H}=2 \times 10^{4} \mathbf{c m}^{-3}$ & $n_{H}=5 \times 10^{3} \mathbf{c m}^{-3}$ & $n_{H}=10^{3} \mathbf{c m}^{-3}$ & \\
\hline $\mathrm{H}_{2} \mathrm{O}$ & $11.23 /-/ 6.55 \times 10^{-06}$ & $53.58 /-/ 5.92 \times 10^{-05}$ & $14.06 /-/ 7.79 \times 10^{-06}$ & $21.27 /-/ 1.57 \times 10^{-06}$ & $20.16 /-/ 2.48 \times 10^{-07}$ \\
\hline HDO & $4.77 / 0.43 / 2.78 \times 10^{-06}$ & $29.00 / 0.54 / 3.20 \times 10^{-05}$ & $28.91 / 2.06 / 1.60 \times 10^{-05}$ & $20.05 / 0.94 / 1.48 \times 10^{-06}$ & $22.50 / 1.12 / 2.77 \times 10^{-07}$ \\
\hline $\mathrm{D}_{2} \mathrm{O}$ & $0.62 / 0.06 / 3.61 \times 10^{-07}$ & $3.40 / 0.06 / 3.76 \times 10^{-06}$ & $7.02 / 0.50 / 3.89 \times 10^{-06}$ & $3.90 / 0.18 / 2.87 \times 10^{-07}$ & $3.38 / 0.17 / 4.15 \times 10^{-08}$ \\
\hline $\mathrm{CH}_{3} \mathrm{OH}$ & $0.88 /-/ 5.15 \times 10^{-07}$ & $4.60 /-/ 5.08 \times 10^{-06}$ & $0.11 /-/ 5.85 \times 10^{-08}$ & $0.04 /-/ 3.19 \times 10^{-09}$ & $-1-1-$ \\
\hline $\mathrm{CH}_{3} \mathrm{OD}$ & $0.31 / 0.36 / 1.83 \times 10^{-07}$ & $1.25 / 0.27 / 1.39 \times 10^{-06}$ & $0.02 / 0.22 / 1.28 \times 10^{-08}$ & $-1-1-$ & $-1-1-$ \\
\hline $\mathrm{CH}_{2} \mathrm{DOH}$ & $0.36 / 0.41 / 2.12 \times 10^{-07}$ & $1.27 / 0.28 / 1.41 \times 10^{-06}$ & $0.03 / 0.33 / 1.92 \times 10^{-08}$ & $-1-1-$ & $-1-1-$ \\
\hline $\mathrm{CHD}_{2} \mathrm{OH}$ & $0.20 / 0.23 / 1.18 \times 10^{-07}$ & $0.51 / 0.11 / 5.60 \times 10^{-07}$ & $0.01 / 0.09 / 5.32 \times 10^{-09}$ & $0.01 / 0.17 / 5.32 \times 10^{-10}$ & $-1-1-$ \\
\hline $\mathrm{CH}_{2} \mathrm{DOD}$ & $0.24 / 0.27 / 1.39 \times 10^{-07}$ & $0.48 / 0.11 / 5.34 \times 10^{-07}$ & $0.02 / 0.16 / 9.58 \times 10^{-09}$ & $-1-1-$ & $-1-1-$ \\
\hline $\mathrm{CD}_{3} \mathrm{OH}$ & $0.09 / 0.10 / 5.27 \times 10^{-08}$ & $0.17 / 0.04 / 1.90 \times 10^{-07}$ & $-/ 0.04 / 2.13 \times 10^{-09}$ & $-1-1-$ & $-1-1-$ \\
\hline $\mathrm{CHD}_{2} \mathrm{OD}$ & $0.08 / 0.09 / 4.42 \times 10^{-08}$ & $0.15 / 0.03 / 1.68 \times 10^{-07}$ & $-/ 0.02 / 1.06 \times 10^{-09}$ & $-1-1-$ & $-1-1-$ \\
\hline $\mathrm{CD}_{3} \mathrm{OD}$ & $0.03 / 0.03 / 1.49 \times 10^{-08}$ & $0.04 / 0.01 / 4.26 \times 10^{-08}$ & $-/ 0.01 / 5.32 \times 10^{-10}$ & $-1-1-$ & $-1-1-$ \\
\hline $\mathrm{CO}_{2}$ & $5.66 /-/ 3.30 \times 10^{-06}$ & $3.31 /-/ 3.65 \times 10^{-06}$ & $0.12 /-/ 6.38 \times 10^{-08}$ & $0.01 /-/ 1.06 \times 10^{-09}$ & $-1-1-$ \\
\hline $\mathrm{CO}$ & $15.20 /-/ 8.86 \times 10^{-06}$ & $0.13 /-/ 1.45 \times 10^{-07}$ & $0.03 /-/ 1.76 \times 10^{-08}$ & $0.04 /-/ 2.66 \times 10^{-09}$ & $-/-1-$ \\
\hline $\mathrm{O}_{2}$ & $5.05 /-/ 2.94 \times 10^{-06}$ & $0.73 /-/ 8.07 \times 10^{-07}$ & $4.72 /-/ 2.61 \times 10^{-06}$ & $0.81 /-/ 5.96 \times 10^{-08}$ & $0.22 /-/ 2.66 \times 10^{-09}$ \\
\hline $\mathrm{O}_{3}$ & $47.90 /-/ 2.79 \times 10^{-05}$ & $0.02 /-/ 1.92 \times 10^{-08}$ & $13.09 /-/ 7.25 \times 10^{-06}$ & $3.22 /-/ 2.37 \times 10^{-07}$ & $0.35 /-/ 4.26 \times 10^{-09}$ \\
\hline $\mathrm{C}$ & $0.03 /-/ 2.02 \times 10^{-8}$ & $0.01 /-/ 1.12 \times 10^{-8}$ & $10.61 /-/ 6.46 \times 10^{-6}$ & $32.89 /-/ 2.42 \times 10^{-6}$ & $31.93 /-/ 3.93 \times 10^{-7}$ \\
\hline $\mathrm{OH}$ & $2.28 /-/ 1.33 \times 10^{-6}$ & $0.04 /-/ 4.26 \times 10^{-8}$ & $2.77 /-/ 1.69 \times 10^{-6}$ & $2.87 /-/ 2.11 \times 10^{-7}$ & $1.87 /-/ 2.29 \times 10^{-8}$ \\
\hline OD & $0.67 / 0.29360 / 3.90 \times 10^{-7}$ & $0.34 / 8.80 / 3.75 \times 10^{-7}$ & $6.70 / 2.42 / 4.08 \times 10^{-6}$ & $4.65 / 1.62 / 3.42 \times 10^{-7}$ & $2.08 / 1.12 / 2.55 \times 10^{-8}$ \\
\hline $\mathrm{CH}_{3}$ & $0.64 /-/ 3.71 \times 10^{-7}$ & $0.66 /-/ 7.25 \times 10^{-7}$ & $8.58 /-/ 5.23 \times 10^{-6}$ & $6.48 /-/ 4.77 \times 10^{-7}$ & $12.03 /-/ 1.48 \times 10^{-7}$ \\
\hline $\mathrm{CH}_{2} \mathrm{D}$ & $0.13 / 0.21 / 7.71 \times 10^{-8}$ & $0.18 / 0.27 / 1.95 \times 10^{-7}$ & $1.38 / 0.16 / 8.40 \times 10^{-7}$ & $0.82 / 0.13 / 6.06 \times 10^{-8}$ & $1.56 / 0.13 / 1.92 \times 10^{-8}$ \\
\hline $\begin{array}{c}\text { No. of } \\
\text { mono-layers }\end{array}$ & 49 & 87 & 50 & 13 & 6 \\
\hline
\end{tabular}

Table 6. Surface coverage, deuterium fractionation ratio and abundance for various sets of binding energies for $n_{H}=2 \times 10^{4} \mathrm{~cm}^{-3}$

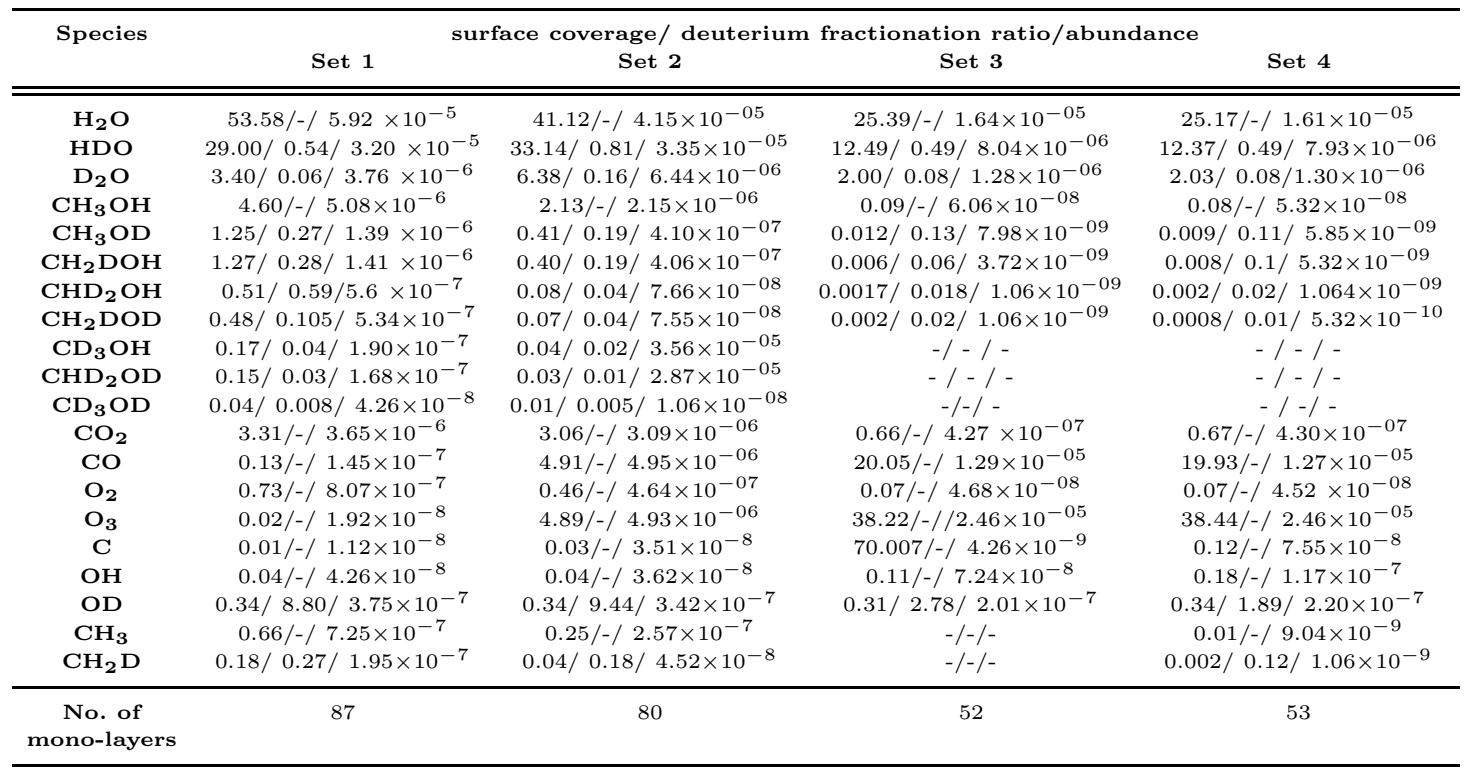

est for atomic hydrogen (see, Table 3 for the energy barriers), hydrogenation reactions are frequent and efficiently produce water as well as methanol. Deuteration reactions are also fast but due to its lower gas phase abundance in comparison to the atomic hydrogen, production of deuterated species are less efficient than the hydrogenated species. In case of Set 2, experimentally obtained energy barriers for olivine grain is used. In this case, atomic hydrogen moves much slower than Set 1 energy barriers, resulting in lower production of hydrogenated species in comparison to the Set 1 energy barrier case. For Set 3, experimental values for the amorphous carbon grain were used and for Set 4, consideration of Lipshtat, Biham \& Herbst (2004) is used. For both the cases, energy barriers against diffusion of hydrogen atom is even higher than Set 2, which results in the insignificant production of methanol. Thus, most of the accreted gas phase CO remain unutilized. Due to the higher desorption energy of $\mathrm{CO}$, it is trapped on the grain and cover significant 
percentage of grain mantles in case of Set 3 and Set $4 . \mathrm{O}_{3}$ occupies a major portion of the grain mantle in case of set 3 and set 4 energy barriers.

\subsection{Effects of irradiation}

Effects of interstellar radiation field on grain mantles were already discussed by Das \& Chakrabarti (2011). They pointed out that the choice of number of affected layers could severely affect the morphology of interstellar grain mantles. Interstellar photons could penetrate deep inside the interstellar ice. So, it is essential to have an idea about the stopping power as well as its range into various ices. In order to see irradiation effects, we study the properties of $0.4 \mathrm{MeV}$ projectiles for various target materials. These calculations are performed by using Zieglers SRIM program (Ziegler 2003). We use $\mathrm{H}^{+}, \mathrm{Fe}^{+}, \mathrm{S}^{+}$and $\mathrm{C}^{+}$as the projectiles. For the target ice, we consider various types of ices shown in Table 6. It is clear from Table 4, that the grain mantle would be mainly covered by $\mathrm{H}_{2} \mathrm{O}, \mathrm{CH}_{3} \mathrm{OH}$, their isotopes and $\mathrm{CO}_{2}$. For one case of Table 6 , we choose, mixed ice as the target ice. Following Das \& Chakrabarti (2011); Majumdar et al. (2012), here, we assume that mixed ice is made up of $70 \%$ water, $20 \%$ methanol and $10 \%$ carbon-di oxide. In Table 6, we have shown the stopping powers (in $\mathrm{MeV} \mathrm{cm}{ }^{2} \mathrm{gm}^{-1}$ ) and projected ranges (in $\mu \mathrm{m}$ ) of these projectiles for various target ices and projectiles. According to theory of transport of ions in matter (TRIM) prescribed by Ziegler (2003), SRIM/TRIM program always makes a guess for the target density by considering a mixture of elemental target densities weighted by their relative stoichiometry. Hudson \& Moore (2004) choose the target density as $1 \mathrm{gm} / \mathrm{cm}^{3}$ for water ice. Since here we compare between various targets under same projectile energy, we keep this density also the same for all the targets. Stopping of ions are different from the weighted stopping of ions in the elemental matter which makes up the compounds (Ziegler 1998). For some target materials this correction is automatically included in the program developed by Ziegler (1998). These corrections are $0.94,0.9470706$ and 1.0 for water, methanol and carbon-di-oxide respectively. Since for the mixed ice no correction was included in the program, we consider it to be 1.0 in our calculation. It is clear from Table 6 that $\mathrm{Fe}^{+}$ has more stopping power than $\mathrm{H}^{+}$but its projected range is lower. However, both the ranges are longer than a typical grain size $(0.1 \mu \mathrm{m})$. So, these projectiles would easily penetrate deep into the ice layers for all of our cases.

\section{CONCLUSIONS}

We carried out Monte Carlo simulation to study chemical composition of interstellar grain mantles under various physical circumstances. We summarize our conclusions bellow:

- Despite low elemental abundance of atomic deuterium in interstellar space, we find that it could significantly alter the chemical composition of grain mantles.

- Binding energies control surface composition of grains. We considered various types of binding energies to study their effects. It is noted that production of surface species is favourable when we use the 'Set 1' energy barriers.
- Interstellar radiation field strongly affects chemical composition of interstellar grain mantles. Our results clearly showed that in diffuse clouds, hardly a few layers of surface species are formed. We also showed that in translucent cloud region, surface coverage of interstellar grain mantles is mainly dominated by photo dissociation products $\left(\mathrm{C}, \mathrm{CH}_{3}, \mathrm{CH}_{2} \mathrm{D}, \mathrm{OH}\right.$ and $\left.\mathrm{OD}\right)$. Around intermediate dense cloud region, water and methanol with their deuterated forms cover major portion of grain mantles. In deep interior (higher density region), formation of oxygenated species $\left(\mathrm{CO}_{2}, \mathrm{O}_{2}, \mathrm{O}_{3}\right)$ increases and significant part of the grain mantles would be covered by $\mathrm{CO}$ molecules as well.

- A comparison between our calculated deuterium fractionation ratio and observed fractionation ratio was made. We obtained a very high degree of deuterium fractionation for water and methanol. Observational evidences also supports this high fractionation feature of methanol but surprisingly, very low fractionation ratio were observed for water molecules. This is in the contradiction with our results. Possible cause of this discrepancy was already discussed by Parise et al. (2003). However, a satisfactory explanation is yet to be found.

- We used $\mathrm{H}^{+}, \mathrm{Fe}^{+}, \mathrm{S}^{+}$and $\mathrm{C}^{+}$ions to study the stopping power and projected range on various types of ice targets such as normal water, deuterated water, normal methanol, deuterated methanol and mixed ice. We find that they do penetrate in the mantles and are likely to heat and evaporate them, apart from causing new ice and ice phase reactions. These will be studied in future.

\section{ACKNOWLEDGMENTS}

$\mathrm{AD}, \mathrm{DS} \& \mathrm{SKC}$ are grateful to ISRO respond (Grant No. ISRO/RES/2/372/11-12), AD \& SKC want to thank DST (Grant No. SB/S2/HEP-021/2013) for financial support. LM thanks MOES and ERC starting grant (3DICE, grant agreement 336474) for funding during this work.

\section{REFERENCES}

Altwegg, K. \& Bockelée-Morvan, D. 2003, Space Sci. Rev., 106, 139 Abelson, P.H., 1966. Proc. Natl. Acad. Sci. 55, 1365.

Allamandola, L. J., Bernstein, M. P., Sandford, S. A., 1997, Astronomica $\&$ Biochemical Origins and the Search for Life in the Universe, ed. Cosmovici, C. B., Bowyer, S., Wertheimer, D., 23, Bologna: Editrice Compositori

Allen, M., Robinson, G. W., 1977., ApJ, 212, 396

Butner, H. M., Charnley, S. B., Ceccarelli, C. et al., 2007, ApJ, 659, L137 Biham, O., Furman, I., Pirronello, V., Vidali, G., 2001, ApJ, 553, 595 Caselli, p., 2002, P\&SS, 50, 1133

Cazaux, S., Cobut, V., Marseille, M., Spaans, M., Caselli, P, A\&A, 522, 74

Caselli, P., Hasegawa, T.I. \& Herbst, E. 1993, ApJ 408, 548

Caselli, P., Ceccarelli, C., 2012, A\&ARv, 20, 56

Ceccarelli, C., Caselli, P., Herbst, E., Tielens, A. G. G. M.; Caux, E., Protostars and Planets V, B. Reipurth, D. Jewitt, and K. Keil (eds.), University of Arizona Press, Tucson, 951, 47

Chang, Q., Cuppen, H., M., Herbst, E., 2005, A\&A, 434, 599

Chakrabarti, S., Chakrabarti, S.K., 2000a. A\&A 354, L6

Chakrabarti, S. K., Chakrabarti, S., 2000b. Ind. J. Phys 74B, 97

Chakrabarti, S.K., Das, A., Acharyya, K.,Chakrabarti, S., 2006, A\&A, 457,167

Chakrabarti, S.K., Das, A., Acharyya, K.,Chakrabarti, S., 2006, BASI, 34 299

Chakrabarti, S. K., Majumdar, L., Das, A., Chakrabarti, S., 2015, Astrophys Space Sci, 357, 90

Charnley, S. B., Tielens, A. G. G. M., \& Rodgers, S. D. 1997, ApJ, 482 L203 


\section{Ankan Das, Dipen Sahu, Liton Majumdar, Sandip K. Chakrabarti}

Table 7. Properties of $0.4 \mathrm{MeV}$ projectile and target material with a target density of $1.0 \mathrm{gm} / \mathrm{cm}^{3}$.

\begin{tabular}{|c|c|c|c|}
\hline Ice & Projectile & $\begin{array}{l}\text { Stopping Power } \\
\left(\mathrm{MeV} \mathrm{cm}^{2} \mathrm{gm}^{-1}\right)\end{array}$ & $\begin{array}{c}\text { Range } \\
(\mu \mathbf{m})\end{array}$ \\
\hline $\mathrm{H}_{2} \mathrm{O} / \mathrm{HDO} / \mathrm{D}_{2} \mathrm{O}$ & $\begin{array}{c}\mathrm{H}^{+} \\
\mathrm{Fe}^{+} \\
\mathrm{S}^{+} \\
\mathrm{C}^{+}\end{array}$ & $\begin{array}{c}425.8 / 403.3 / 383.0 \\
1905 / 1804 / 1714 \\
2819 / 2669 / 2535 \\
2875 / 2722 / 2585\end{array}$ & $\begin{array}{c}6.68 / 7.06 / 7.43 \\
0.6253 / 0.6731 / 0.7230 \\
0.9569 / 1.03 / 1.11 \\
1.86 / 1.98 / 2.11\end{array}$ \\
\hline $\mathrm{CH}_{3} \mathrm{OH} /$ singly deuterated/doubly deuterated & $\begin{array}{c}\mathrm{H}^{+} \\
\mathrm{Fe}^{+} \\
\mathrm{S}^{+} \\
\mathrm{C}^{+}\end{array}$ & $\begin{array}{c}461.1 / 447.1 / 433.8 \\
2591 / 2512 / 2438 \\
3678 / 3566 / 3461 \\
3427 / 3322 / 3224\end{array}$ & $\begin{array}{c}6.09 / 6.28 / 6.48 \\
0.5648 / 0.5881 / 0.6118 \\
0.8117 / 0.8451 / 0.8791 \\
1.52 / 1.58 / 1.63\end{array}$ \\
\hline $\mathrm{CO}_{2}$ & $\begin{array}{c}\mathrm{H}^{+} \\
\mathrm{Fe}^{+} \\
\mathrm{S}^{+} \\
\mathrm{C}^{+}\end{array}$ & $\begin{array}{l}364.8 \\
1890 \\
2712 \\
2679\end{array}$ & $\begin{array}{c}8.28 \\
0.7225 \\
1.07 \\
2.01\end{array}$ \\
\hline Mixed ice & $\begin{array}{c}\mathrm{H}^{+} \\
\mathrm{Fe}^{+} \\
\mathrm{S}^{+} \\
\mathrm{C}^{+}\end{array}$ & $\begin{array}{l}445.4 \\
3425 \\
3186 \\
3138\end{array}$ & $\begin{array}{c}6.42 \\
0.6151 \\
0.9075 \\
1.71\end{array}$ \\
\hline
\end{tabular}

Charnley, S.B., Tielens, A.G.G.M. \& Millar, T. J. 1992, ApJ, 399, L71

Coutens A., Vastel C., Cazaux S. et al., 2013, A\&A, 553, A75

Cuppen, H., Herbst, E., 2007, ApJ, 668,294

Dartois, E., Schutte, W., Geballe, T. R., et al. 1999, A\&A, 342, L32

Dartois, E., Thi, W.-F., Geballe, T. R., et al. 2003, A\&A, 399, 1009

Das, A., Acharyya, K., Chakrabarti, S. \& Chakrabarti, S. K.,2008b, A\&A, 486,209

Das, A., Chakrabarti, S. K., Acharyya K. \& Chakrabarti, S., 2008a, NEWA, 13, 457

Das, A., Acharyya, K. \& Chakrabarti, S. K., 2010, MNRAS 409, 789

Das, A. \& Chakrabarti, S. K., 2011, 418. 545, MNRAS

Das, A. Majumdar, L., Chakrabarti, S. K., \& Chakrabarti S., 2013, New Astronomy, 23, 118

Das, A. Majumdar, L., Chakrabarti, S. K., \& Chakrabarti S., 2013, MNRAS, 433, 3152

Das, A., Majumdar, L., Sahu, D., Gorai, P., Sivaraman, B., Chakrabarti, S. K., 2015, 808, 21

Das, A., Majumdar, L., Chakrabarti, S. K., \& Sahu, D., NEWA, 2015, 35, 53

Draine, B. T., 2003, APJ 598, 1017

Ehrenfreund, P., Charnley, S. B., 2000, ARAA, 38,427

Fuchs, G. W., Cuppen, H. M., Ioppolo, S. et al., 2009, A\&A, 505, 629

Flower, D. R., Forets, G. Peineau des, Walmsley, C. M., 2006, A\&A, 449, 621

Gensheimer, P. D., Mauersberger, R., \& Wilson, T. L. 1996, A\&A, 314, 281

Gibb, E. L., Whittet, D. C. B., Boogert, A. C. A., Tielens, A. G. G. M., 2004, ApJS 151, 35

Greenberg J. M., 1999, Formation and Evolution of Solids in Space, ed. Greenberg, J. M., Li, A., 53, Dordrecht: Kluwer

Herbst, E., van Dishoeck, E. F., 2009, ARA\&A, 47, 427

Hudson, R. L., Moore, M. H., 2004, ICARUS, 172, 466

J $\phi$ rgensen J. K., van Dishoeck E. F., 2010, ApJ, 725, L172

Kaiser R. I., Roessler, K., 1998. ApJ, 503, 959

Katz, N., Furmann, I., Biham, O., Pironello, V. and Vidali, G., 1999, ApJ 522,305

Lipshtat, A., Biham, O., \& Herbst, E., 2004, MNRAS, 348, 1055

Linsky, J.L., Diplas, A., Wood, B.E., Brown, A., Ayres, T.R., Savage,

B.D., 1995, ApJ. 451, 335, B351

Linsky, J. L., 2003, SSRv, 106, 49

Loinard, L., Castets, A., Ceccarelli, C., Caux, E., Tielens, A. G. G. M., 2001, ApJ, 552, L163

Liu, F., Parise, B., Kristensen, L., et al. 2011, A\&A, 527, A19

Majumdar, L., Das, A., Chakrabarti, S.K., 2014, A\&A, 562, A56

Majumdar, L., Das, A., Chakrabarti, S.K., 2014, ApJ, 782, 73

Majumdar, L., Das, A., Chakrabarti, S.K., Chakrabarti, S., 2013, New Astronomy, 20, 15

Majumdar, L., Das, A., Chakrabarti, S.K., Chakrabarti, S., 2012, Research in Astronomy \& Astrophysics, 12, 1613

Moore, M. H., Hudson R. L,. 1992. ApJ, 401, 353

Orgel, L.E., 2004. Biochem. Mol. Biol. 39, 99

Osamura, Y., Roberts, H., Herbst, E., 2004, A\&A, 421, 1101

Parise, B., Ceccarelli, C., Tielens, A. G. G. M., et al. 2002, A\&A, 393, L49

Parise, B., Simon, T., Caux, E., Dartois, E., Ceccarelli, C., Rayner, J., \&

Tielens, A.G.G.M. 2003, A\&A, 410, 897
Parise, B., Castets, A., Herbst, E., et al. 2004, A\&A, 416, 159

Parise, B., Ceccarelli, C., Maret, S., 2005, A\&A, 441, 171

Persson, M. V., Jørgensen, J. K., van Dishoeck, E. F., 2012, 541, 39

Pirronello, V., Biham, O., Liu, C., Shena, L. and Vidali, G., 1997, ApJ,

$483 \mathrm{~L}, 131$

Pirronello, V., Liu, C., Riser, J.E. and Vidali, G., 1999, A\&A, 344, 681

Pontoppidan, K. M., Dartois, E., van Dishoeck, E. F., Thi, W.-F., \& dHendecourt, L. 2003, A\&A, 404, L17

Roueff, E., Tiné, S., Coudert, L. H., et al. 2000, A\&A, 354, L63

Sahu, D., Das, A., Majumdar, L., Chakrabarti, S. K., 2015, New Astron., doi:10.1016/j.newast.2014.12.011

Schmitt, B., 1994, Molecules and Grains in Space, ED. Nenner, I., 735, New York: AIP Press

Spaans, M., Ehrenfreund, P., 1999, Laboratory Astrophysics and Space Research, ed. Ehrenfreund, P., Krafft, K., Kochan, H., Pironello, V., 1, Dordrecht:kluwer

Snow, T. P., McCall, B. J., 2006, ARAA, 44, 367

Stantcheva, T., Herbst, E., 2004, A\&A, 423, 241

Stancil, P. C., Lepp, S., Dalgarno, A., 1998, ApJ, 509,1

Tielens, A.G.G.M., Hagen, W., 1982, A\&A, 114, 245

Taquet V., Lpez-Sepulcre A., Ceccarelli C., Neri R., Kahane C., Coutens A., Vastel C., 2013, ApJ, 768, L29

Turner, B. E., 1990, ApJ, 362, 29

van der Tak, F. F. S., \& van Dishoeck, E. F. 2000, A\&A, 358, L79

van der Tak, F. F. S., Schilke, P., Müller, H. S. P., Lis, D. C., Phillips, T. G., Gerin, M., Roueff, E., 2002, A\&A, L53

Wakelam, V., Vastel, C., Aikawa, Y., Coutens, A., Bottinelli, S., \& Caux, E., 2014, MNRAS, 445(3), 2854

Whittet, D. C. B., Pendleton, Y. J., Gibb, E. L., Boogert, A. C. A., Chiar, J. E., \& Nummelin, A. 2001, ApJ, 550, 793

Whittet, D. C. B. 2003, Dust in the Galactic Environment (Bristol: Inst. Phys. Publ.)

Ziegler, J. F. 2003, Manoyan, J. M., Nucl. Inst. Methods, 1998, B35, 215 Ziegler, J. F. 2003, Stopping and Range of Ions in Matter SRIM2003 (available www.srim.org) 


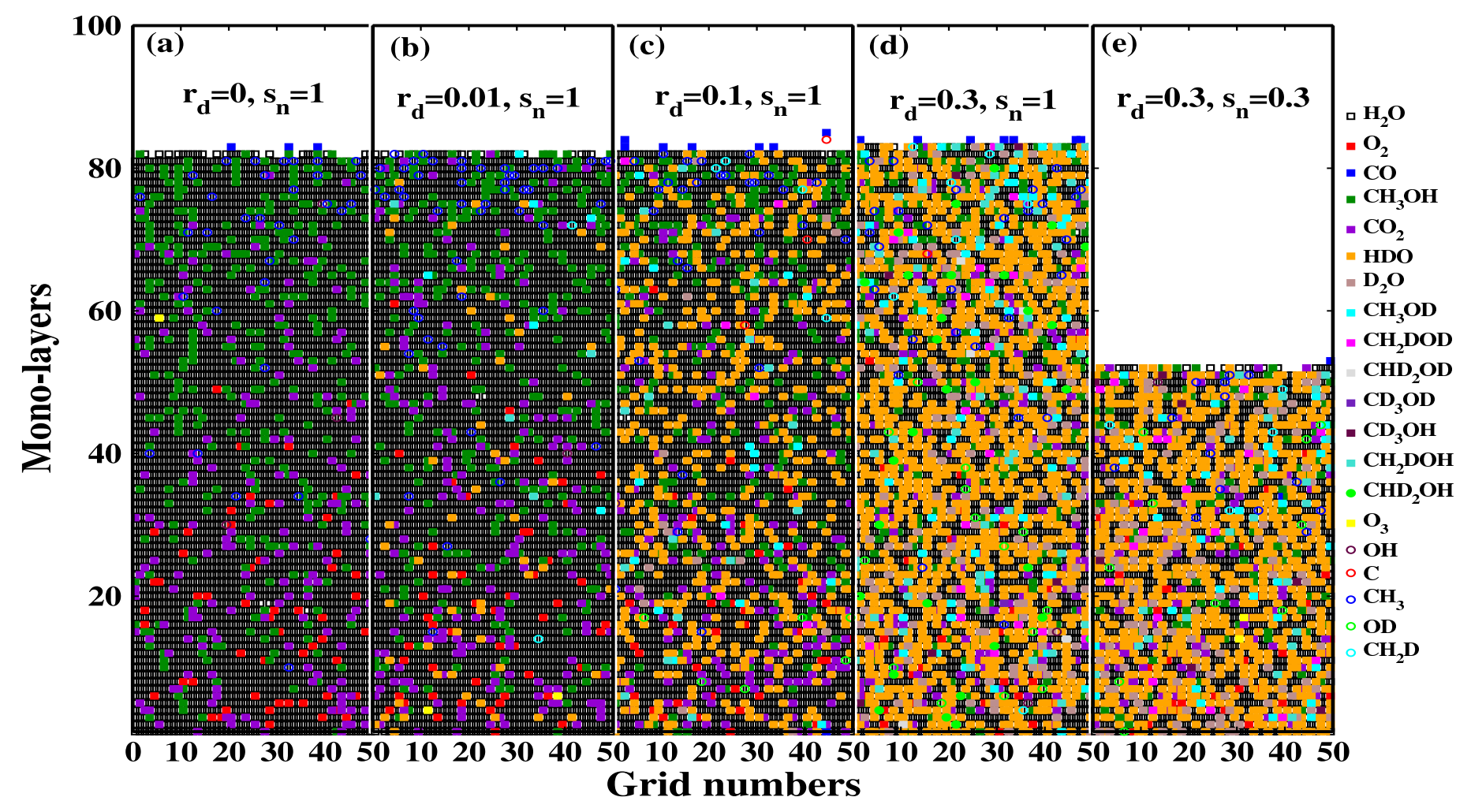

Figure 2. (a-e) A cross-sectional view of the composition of grain mantle with the (a) absence and (b-e) presence of deuterium. 


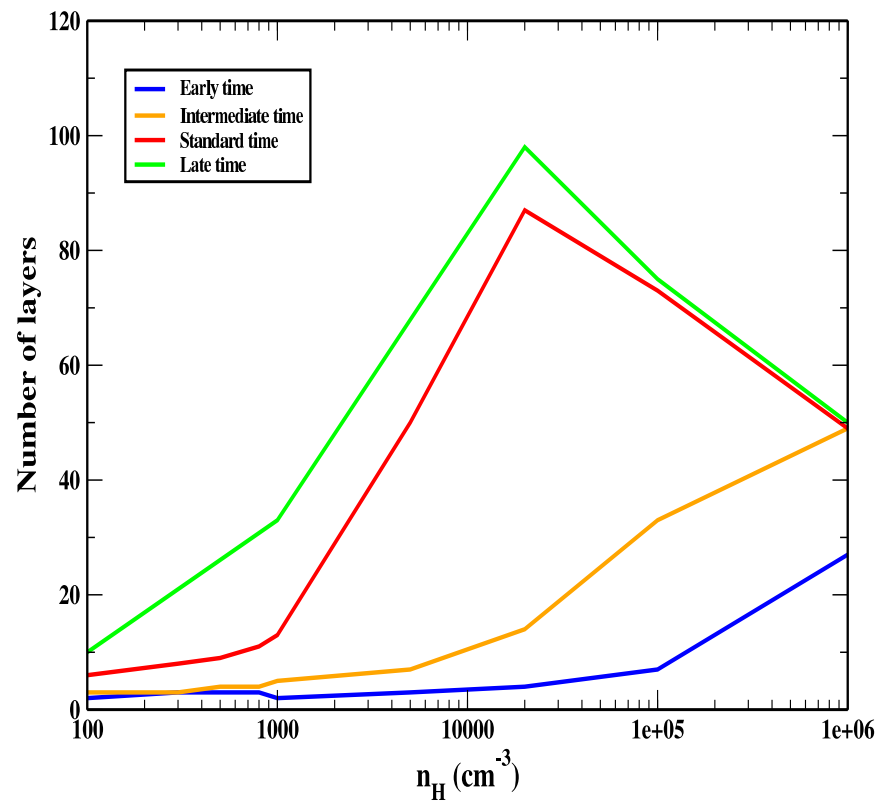

Figure 3. Formation of numbers of layers in various stages of chemcal evolution. 


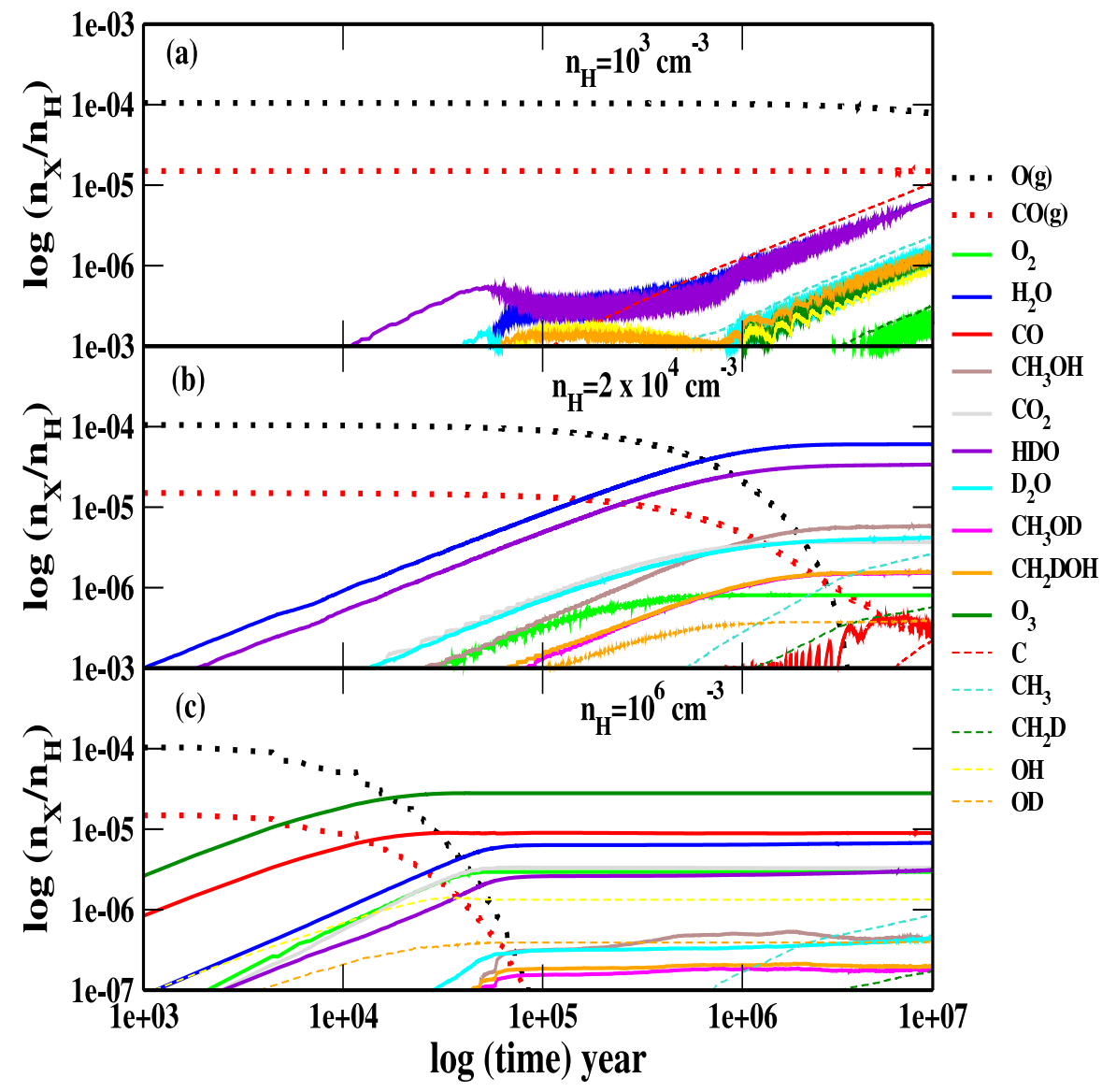

Figure 4. (a-c) Chemical evolution of important gas/ice phase species for (a) $n_{H}=10^{4} \mathrm{~cm}^{-} 3$, (b) $n_{H}=10^{5} \mathrm{~cm}^{-3}$ and (c) $n_{H}=$ $10^{6} \mathrm{~cm}^{-3}$. 


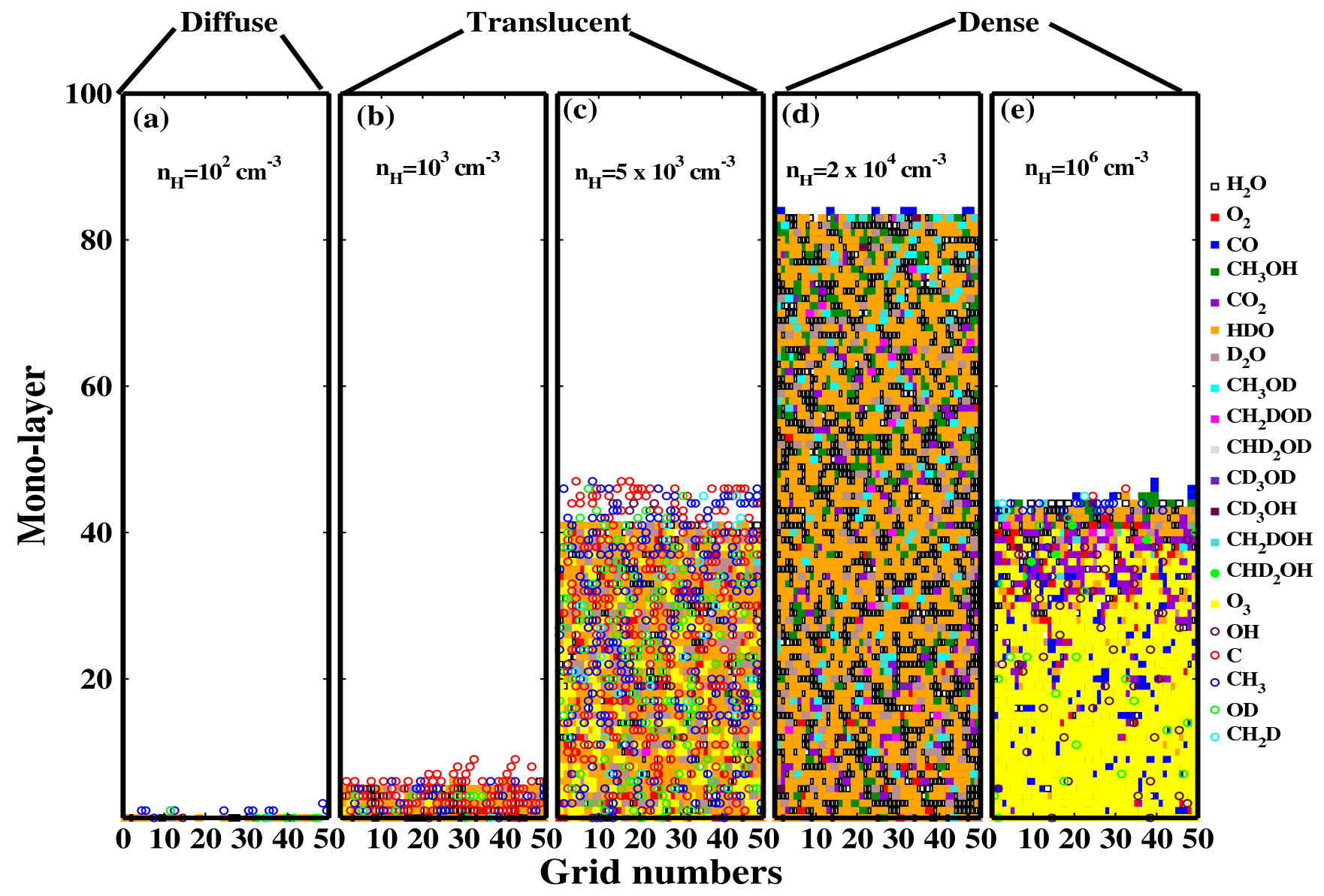

Figure 5. (a-e) Composition of grain mantle for various types of clouds. 


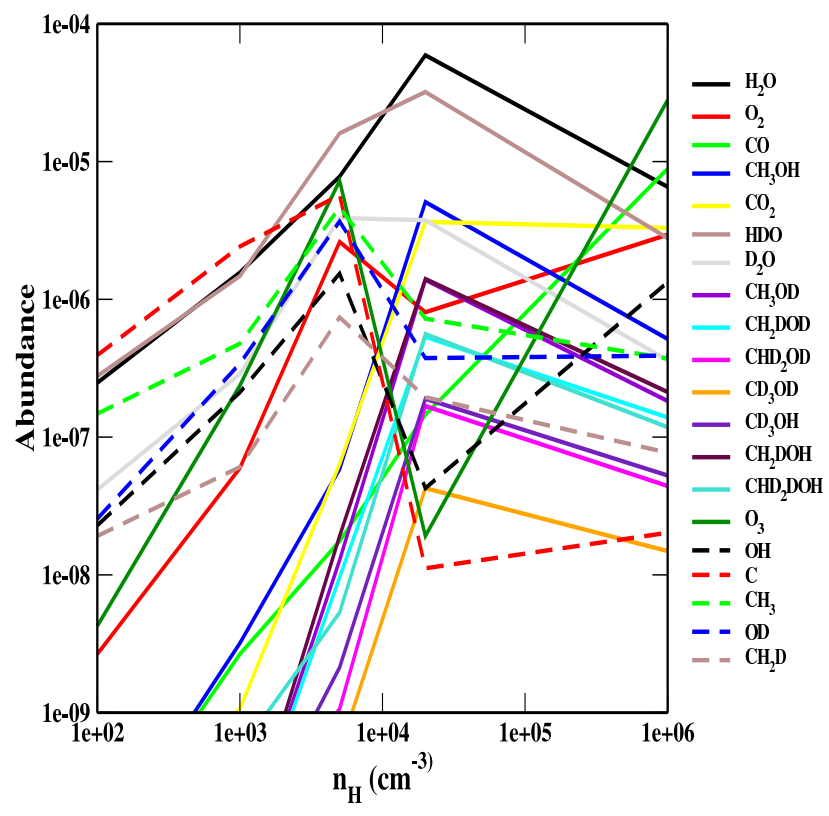

Figure 6. Abundances of some important surface species around various clouds. 


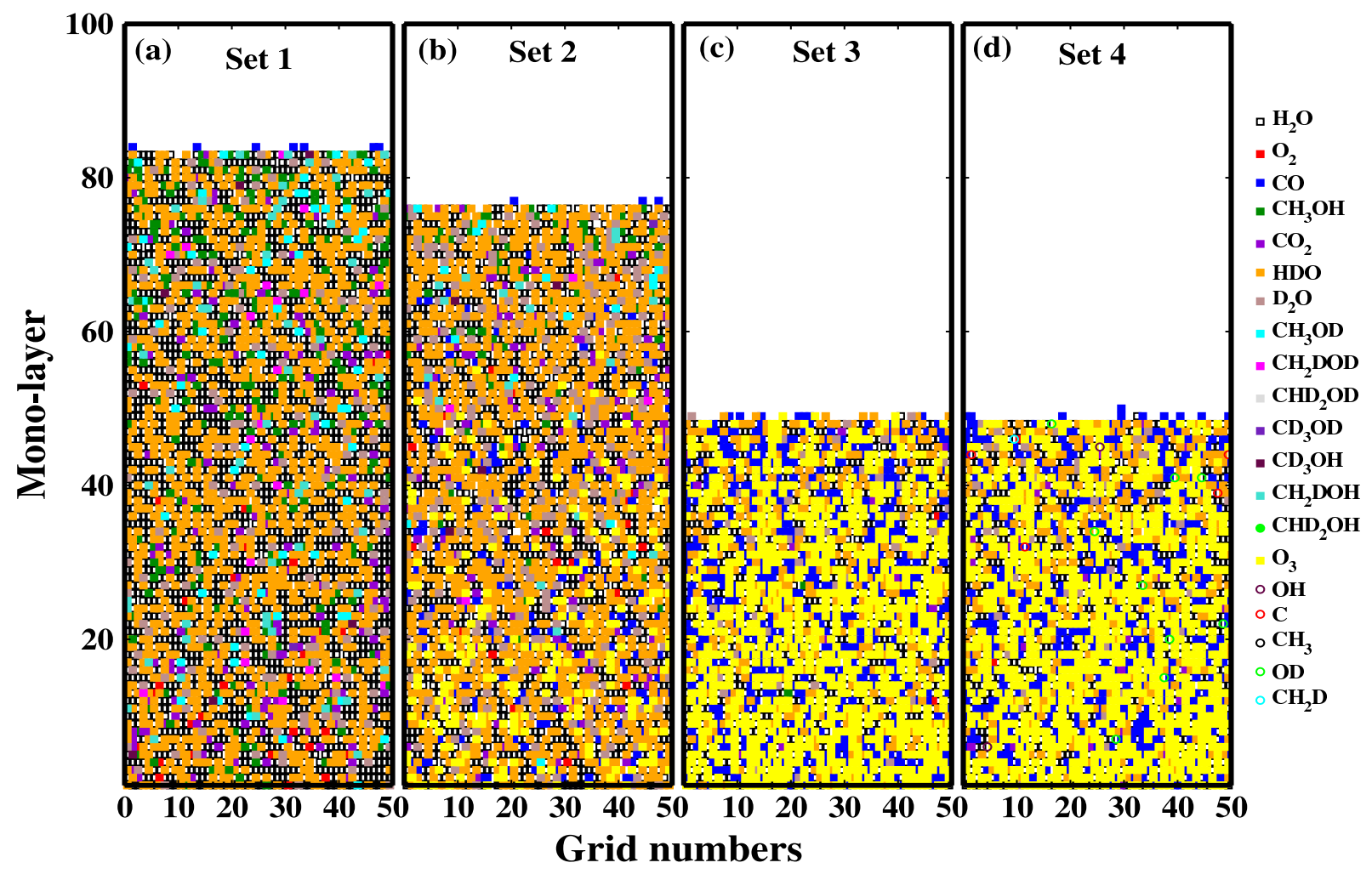

Figure 7. (a-d) Composition of grain mantle for various sets of binding energies. 